\title{
Article \\ DisA Restrains the Processing and Cleavage of Reversed Replication Forks by the RuvAB-RecU Resolvasome
}

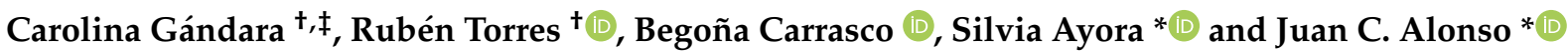 \\ Department of Microbial Biotechnology, Centro Nacional de Biotecnología, CNB-CSIC, 3 Darwin St, \\ 28049 Madrid, Spain; carolina.rosa@gmail.com (C.G.); rtorres@cnb.csic.es (R.T.); bcarrasc@cnb.csic.es (B.C.) \\ * Correspondence: sayora@cnb.csic.es (S.A.); jcalonso@cnb.csic.es (J.C.A.) \\ † These authors contributed equally to this work. \\ $\ddagger$ Present address: Newcells Biotech Ltd., Newcastle-upon-Tyne NE4 5BX, UK.
}

Citation: Gándara, C.; Torres, R.; Carrasco, B.; Ayora, S.; Alonso, J.C. DisA Restrains the Processing and Cleavage of Reversed Replication Forks by the RuvAB-RecU Resolvasome. Int. J. Mol. Sci. 2021, 22, 11323. https:// doi.org/10.3390/ijms222111323

Academic Editor: David Alexander Forsyth Gillespie

Received: 31 August 2021

Accepted: 16 October 2021

Published: 20 October 2021

Publisher's Note: MDPI stays neutral with regard to jurisdictional claims in published maps and institutional affiliations.

\begin{abstract}
DNA lesions that impede fork progression cause replisome stalling and threaten genome stability. Bacillus subtilis RecA, at a lesion-containing gap, interacts with and facilitates DisA pausing at these branched intermediates. Paused DisA suppresses its synthesis of the essential c-di-AMP messenger. The RuvAB-RecU resolvasome branch migrates and resolves formed Holliday junctions (HJ). We show that DisA prevents DNA degradation. DisA, which interacts with RuvB, binds branched structures, and reduces the RuvAB DNA-dependent ATPase activity. DisA pre-bound to HJ DNA limits RuvAB and RecU activities, but such inhibition does not occur if the RuvAB- or RecU-HJ DNA complexes are pre-formed. RuvAB or RecU pre-bound to HJ DNA strongly inhibits DisA-mediated synthesis of c-di-AMP, and indirectly blocks cell proliferation. We propose that DisA limits RuvAB-mediated fork remodeling and RecU-mediated $\mathrm{HJ}$ cleavage to provide time for damage removal and replication restart in order to preserve genome integrity.
\end{abstract}

Keywords: replication stress; DNA damage signal; fork reversal; c-di-AMP; RuvAB; RecU; DisA

\section{Introduction}

In living cells, replication fork progression is frequently hindered by obstacles in and on the DNA template [1-4]. Cells may use several strategies when DNA replication is challenged by this stress: replication forks stall, DNA polymerases uncouple, lesions can be simply skipped by the replisome forming single-stranded DNA (ssDNA) gaps, or the stalled fork is pushed backwards to convert it into a Holliday junction (HJ)-like structure by allowing the pairing of the two nascent strands and rewinding of the parental strands (fork reversal, known also as fork regression) [5-9]. Nevertheless, in Escherichia coli, fork reversal appears to be a less relevant response to DNA damage, and is infrequent in wild-type ( $w t)$ cells, because reversed forks are susceptible to nucleolytic degradation of the regressed nascent DNA arms. In fact, RecBCD (counterpart of Bacillus subtilis AddAB) prevents or removes reversed fork structures, and in the $\triangle \operatorname{rec} B C D$ context, the reversed forks are processed by the RuvAB translocase and cleaved by the RuvC (counterpart of $B$. subtilis RecU) $\mathrm{HJ}$ resolvase, leading to fork breakage and one-ended double-strand breaks (DSBs) $[7,8,10]$. In sharp contrast, in mammalian cells [11] or during the early stage of $B$. subtilis spore revival $[12,13]$, dedicated mechanisms are actively involved in the formation and integrity of reversed forks. Therefore, the analysis of the repair functions active in reviving B. subtilis spores gives clues of the proteins that contribute to genome integrity after fork stalling, because breakage of a reversed fork should be pathological during phases where only one genome copy is available, and cells should prevent it (unless stated otherwise, indicated genes and products are of B. subtilis origin).

When the DNA of an inert mature haploid spore is damaged by ionizing radiation, and then the spores are synchronously revived under unperturbed conditions, spores lacking both $\mathrm{Add} A B$ and RecJ exonucleases are as capable of repairing the damage as 
the $w t$ control [12], showing that long-range end-resection functions play a minor role in removing a replicative stress. Indeed, these proteins are synthesized after initiation of spore replication and prior to cell elongation $[14,15]$. By contrast, spores required the recombinase RecA, its mediators and modulators, the branch migration translocases (RuvAB, RecG), the $\mathrm{HJ}$ resolvase $\mathrm{RecU}$, and the DNA damage checkpoint sensor DisA for replication fork rescue $[12,13]$. We can envision that during the first replication cycle of those reviving spores, in the absence of an intact homologous template and end-resection functions, the fork may be reversed to remove a replicative stress. This way, the lesion is placed on a double-stranded (ds) configuration to permit its removal by means of base- and nucleotideexcision repair. Then, the regressed nascent complementary DNA arms can potentially allow for the bypass of template DNA obstructions by error-prone and error-free DNA damage tolerance (DDT) sub-pathways that contribute to bypass or circumvent the lesion. Nevertheless, fork remodeling could be controlled by a poorly characterized function to avoid the formation of highly detrimental DSBs.

In vivo analyses showed that DisA is a DNA sensor protein found in the Firmicutes and Actinobacteria phyla, among others [16]. DisA forms a focus that moves rapidly along the chromosome scanning for "perturbations" but pauses its movement upon DNA damage and delays entry into sporulation until the offending lesion is removed [17]. DisA still pauses in the absence of long-range end resection ( $\triangle a d d A B \triangle r e c J$ cells) [18], but it does not pause in the absence of RecO and RecA. This suggests that DisA pauses at a DNA structure formed after $\mathrm{RecO}$ and RecA engagement at a site of replicative stress, so that the primary signal recognized by DisA is a lesion-containing gap or a branched intermediate rather than DNA ends or DSBs. While scanning the chromosome, DisA converts a pair of ATPs into the essential second messenger cyclic $3^{\prime}, 5^{\prime}$-diadenosine monophosphate (c-di-AMP) [19]. Paused DisA, however, drops the synthesis of c-di-AMP in response to replication perturbations, as those induced by methyl methane sulfonate (MMS) [20], to levels comparable to that in the absence of DisA [21]. DisA is composed of an N-terminal globular domain featuring diadenylate cyclase (DAC) activity, and a C-terminal RuvA-like HJ DNA-binding domain, separated by a central helical domain [22]. Octameric DisA, upon binding to a branched DNA structure, undergoes a conformational change that inhibits its c-di-AMP synthesis [22-24]. Low c-di-AMP levels indirectly increase (p)ppGpp synthesis [25], and (p)ppGpp blocks DNA replication by inhibiting DNA primase activity [26]. This DisA fail-safe mechanism of coordinating repair-by-recombination and replication is indirect, because DisA neither compromised PriA-dependent initiation of DNA replication nor affected replication elongation using an in vitro reconstituted replication assay [13].

DisA acts selectively at stalled forks, since $\triangle$ dis $A$ cells show a similar resistance to that of $w t$ cells upon exposure to $\mathrm{H}_{2} \mathrm{O}_{2}$ or nalidixic acid, which induce single-strand nicks or DSBs, respectively [21,23]. Single-molecule studies revealed that DisA shows a dynamic movement in exponentially growing $w t$ cells, but it becomes static when branched recombination intermediates accumulate, as in the absence of the RecU HJ resolvase or the RecG branch migration translocase [23]. The current model for DisA action is that this sensor protein may control the response to a replicative stress until the damage is removed, and balances the benefits and risks of fork remodeling $[13,17,18,27]$. It is poorly understood whether functions that remodel branched DNA structures affect c-di-AMP synthesis.

One may question which function(s) remodel a stalled replication fork in B. subtilis. In E. coli, stalled forks are processed by the RecA, RecG, RuvAB, or RecQ remodelers [28-32] or the ssDNA gap left by the skipped lesion is extended by RecJ and RecQ to facilitate RecA-mediated gap repair [7,28-32]. Among the functions required for B. subtilis spore survival upon DNA damage are $\operatorname{Rec} A, \operatorname{RuvAB}, \operatorname{Rec} \mathrm{U}, \operatorname{RecG}$, and DisA, and genetic data have shown that the dis $A$ gene is epistatic to $\operatorname{rec} A, \operatorname{ruv} A, \operatorname{ruv} B, \operatorname{rec} U$, and $\operatorname{rec} G$ genes upon exposure to ionizing radiation $[12,13]$. This suggests that DisA acts with these proteins in common mechanisms to ensure the stability of the stalled forks and the maintenance of cell survival. Previous studies have explored how DisA could modulate RecA or RecG 
activities $[18,27,33]$, but the interplay between DisA and the resolvasome (RuvAB-RecU) is poorly understood.

When a replicative stress occurs, the RuvAB levels increase as part of the SOS response, and the DisA and RecU pools increase as part of the cell envelope stress response [34-36], suggesting a temporal link between increasing levels of these proteins and a replication perturbation. RuvAB and $\mathrm{Rec} U$ are the B. subtilis counterpart of the E. coli RuvABC $\left(\right.$ RuvABC$_{E c o}$ ) resolvasome [37-41]. RuvA and RuvB are among the most ubiquitous bacterial proteins, whereas RecU is selectively found in bacteria of the Firmicutes and Tenericutes phyla. The RecU structure, which is unrelated to RuvC, shares homology to certain bacteriophages and archaeal $\mathrm{HJ}$-resolving enzymes [42,43]. The resolvasome might act at reversed forks (HJs) in response to a stalled fork and is crucial for the processing of double HJs during canonical DSB repair [5-7]. RuvA specifically binds and stabilizes HJs [37]. A RuvA-HJ complex is the first crucial step for RuvB loading and for the formation of an ATP-dependent RuvAB motor [44,45]. RuvB interacts with RecU [36]. RecU specifically binds HJ DNA [46,47]. Finally, once RuvAB-mediated branch migration exposes the RecU cognate site, RecU cleaves the HJ to yield two nicked duplexes $[46,48]$.

Taking the in vivo data into account, in this study, we biochemically explored how DisA could modulate the stability of DNA structures that mimic a stalled or reversed replication fork, by analyzing its interplay with RuvAB and RecU. We show that DisA contributes to reducing chromosome degradation. DisA, which binds HJ DNA with high affinity in the presence of physiological $\mathrm{Mg}^{2+}$ concentrations, physically interacts with RuvB. RuvAB branch migrates a fixed (HJ-J3) or mobile (HJ-J4) DNA to restore a replication fork, but it poorly converts a stalled fork into an HJ-like structure. DisA inhibits RuvABmediated ATP hydrolysis and processing of HJs. DisA, which does not interact with RecU, inhibits RecU-mediated resolution of HJs. In the presence of RuvAB or RecU bound to HJ-J3 DNA, DisA-mediated c-di-AMP synthesis is strongly inhibited. These data suggest that DisA may ensure fork stability by timely coordinating RuvAB- and RecU-mediated processing of branched intermediates at the damaged replication fork.

\section{Results}

\subsection{DisA Preferentially Binds DNA at High $\mathrm{Mg}^{2+}$ Concentrations}

Single-molecule studies revealed that: (i) the dynamic movement of DisA pauses at a RecA-bound lesion-containing gap in sporulating cells [18]; (ii) DisA becomes static in the $\Delta r e c U$ context, where branched intermediates accumulate [23]; and (iii) DisA-mediated c-di-AMP synthesis is unaffected by duplex DNA but is inhibited by branched intermediates [22]. Thus, it is likely that octameric DisA, which consists of two peripheral quartets of helix-hairpin helix $(\mathrm{HhH})$ domains connected to the central DAC domains by a helical spine [22], preferentially binds to stalled or reversed forks in a universe of duplex chromosomal DNA through its HhH domains that resemble that of RuvA. To characterize the specific DNA structure recognized by DisA, DNA binding was analyzed by electrophoretic mobility shift assays (EMSAs). The addition of $0.2 \%$ glutaraldehyde prior to separating the DisA-DNA complexes, however, was necessary to detect and visualize them.

In the presence of physiological $\mathrm{Mg}^{2+}$ concentrations, DisA bound both dsDNA and HJ-J3 DNA with similar apparent DNA binding constant $\left(\mathrm{K}_{\text {Dapp }}\right)$ values $(2.6 \pm 0.3 \mathrm{nM}$ and $3.0 \pm 0.5 \mathrm{nM}$, respectively), but the complexes formed with these two substrates were different (Figure 1A,B). A fast-moving complex was observed with dsDNA, whereas DisA$\mathrm{HJ}-\mathrm{J} 3$ complexes tend to remain trapped in the well even at low acrylamide concentrations, suggesting that the RuvA-like $\mathrm{HhH}$ tetrameric domains arranged at each side of the dumbbell-shaped DisA octameric structure interact differently with duplex and HJ-J3 DNA [22]. DisA also bound flayed DNA (unreplicated fork DNA) and single-stranded (ss) DNA with a similar high affinity ( $\mathrm{K}_{\text {Dapp }} 3.2 \pm 0.2 \mathrm{nM}$ and $3.3 \pm 0.1 \mathrm{nM}$, respectively) (Supplementary Materials Figure S1A), and formed complexes that remained trapped in the well (data not shown). This unexpected result suggests that (an) auxiliary protein(s) or 
cofactor specifically recruits DisA to the stalled fork, or HJ DNA. Alternatively, we did not use the proper conditions for DisA.

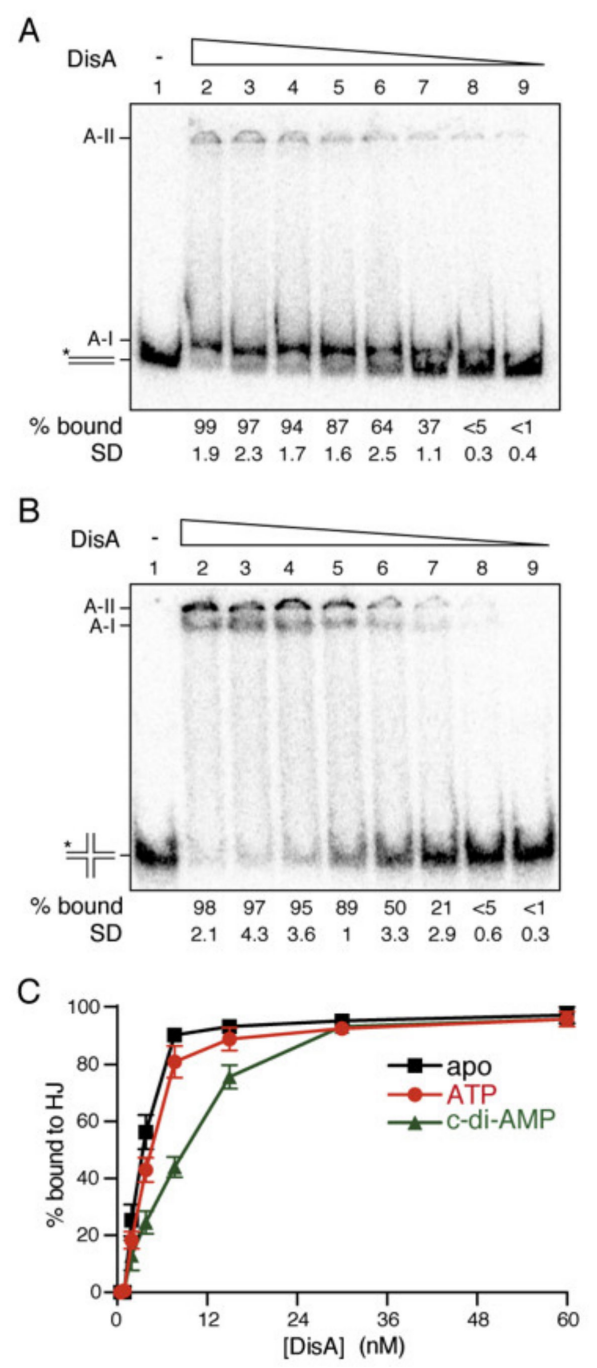

Figure 1. DisA binding to DNA. $\left[\gamma_{-}{ }^{32} \mathrm{P}\right]$ dsDNA (A) or $\left[\gamma^{32} \mathrm{P}\right] \mathrm{HJ}$ DNA (B) was incubated with increasing DisA concentrations (doubling from 0.45 to $60 \mathrm{nM}$ ) in buffer $\mathrm{C}$ containing $10 \mathrm{mM} \mathrm{MgCl}_{2}$, and after fixation, complexes were detected by EMSA. Experiments were repeated at least 3 times. A representative gel and below the mean \% of DNA bound and its SD are shown. A-I denotes complexes entering the gel and A-II denotes complexes retained in the well. (C) ATP or c-di-AMP barely affects DisA binding to HJ DNA. $\left[\gamma_{-}^{-32} \mathrm{P}\right] \mathrm{HJ}$ DNA was incubated with increasing DisA concentrations $\left(15 \mathrm{~min}\right.$ at $37^{\circ} \mathrm{C}$ ) in buffer $\mathrm{C}$ containing $10 \mathrm{mM} \mathrm{MgCl}_{2}$ or $10 \mathrm{mM} \mathrm{MgCl}_{2}$ and $500 \mu \mathrm{M}$ ATP or c-di-AMP $\left(15 \mathrm{~min}\right.$ at $\left.37^{\circ} \mathrm{C}\right)$. The complexes, detected by EMSA, were quantified. Results are shown as the mean \pm SEM of $>3$ independent experiments.

To test the latter hypothesis, we searched for the optimal conditions for the DAC activity of DisA. DisA-mediated c-di-AMP synthesis was not detected $(p<0.01)$ in the absence of $\mathrm{Mg}^{2+}$ ions (presence of $5 \mathrm{mM}$ EDTA). In the presence of $1 \mathrm{mM} \mathrm{MgCl}_{2}$, DisA poorly catalyzed c-di-AMP synthesis, whereas the DAC activity of DisA was significantly increased in the presence of $10 \mathrm{mM} \mathrm{MgCl}$ (Figure S2A). In the presence of $10 \mathrm{mM} \mathrm{MgCl}$, DisA-mediated c-di-AMP synthesis was inhibited by the addition of HJ-J3 DNA $(p<0.01)$ but not by dsDNA $(p>0.1)$ (Figure S2B) [22]. Similarly, binding to DNA was best at high than at low $\mathrm{MgCl}_{2}$ concentrations or in the presence of EDTA (Annex 1, Figure S1A,B). It is likely that in the presence of physiological $\mathrm{Mg}^{2+}$ concentrations $(10 \mathrm{mM})$, DisA forms a stable macro-complex with HJ-J3 DNA that is not entering in a gel and suppresses its DAC 
activity. DisA, however, forms only a transient interaction with duplex DNA, which was captured in our EMSA assays because of the use of glutaraldehyde. Thus, the presence of dsDNA does not affect DisA-mediated DAC activity (Figure S2).

Finally, the effect of nucleotide cofactors in HJ-J3 binding was tested. DisA, in the ATP-bound form, bound HJ-J3 DNA with slightly less affinity ( $K_{\text {Dapp }} 5.5 \mathrm{nM} \pm 0.3 \mathrm{nM}$ ) (Figure 1C). Saturating c-di-AMP concentrations further reduced the interaction of DisA with HJ-J3 DNA (Figure 1C), suggesting that when DisA converts ATP into c-di-AMP, this interferes with HJ DNA binding. It is likely that: (i) DisA interacts transiently and dynamically with duplex chromosomal DNA, and such interaction is not sufficient to suppress DisA-mediated c-di-AMP synthesis (Figures 1A and S2); (ii) DisA bound to branched intermediates (e.g., a stalled or reversed fork) forms a large molecular mass complex that undergoes a conformational change to suppress DisA-mediated c-di-AMP synthesis (Figures 1B and S2); and (iii) (an) unknown protein(s) might contribute to recruit and stabilize DisA at branched intermediates, with RecA, which physically interacts with DisA [18], being a good candidate to help DisA loading.

\subsection{DisA Interacts with RuvB}

A genetic interaction of DisA with the branch migration translocases (RuvAB or RecG) in response to DNA damage was inferred from survival assays [13]. The RecG enzyme fails to form stable complexes with DisA [27]. Here, we analyzed if RuvAB interacts with DisA. To test this, a bacterial two-hybrid system was used (see the material and methods) (Figure 2A-C).

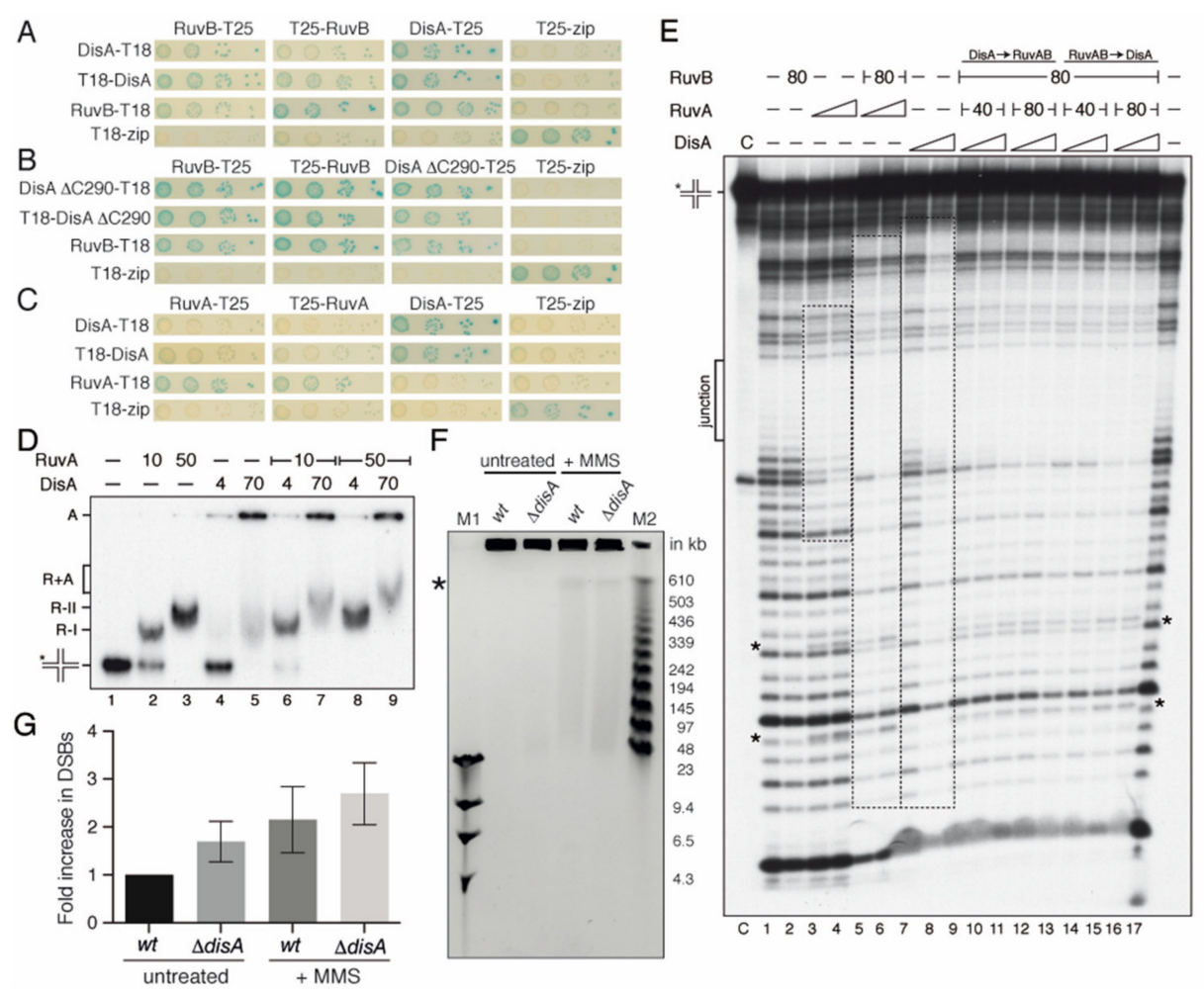

Figure 2. DisA interacts with RuvB and may contribute to fork protection. (A-C) Bacterial two-hybrid interaction assays were done, co-transforming the pair of plasmids expressing full-length DisA, DisA $\Delta$ C290, RuvB, or RuvA fused at the N- or C-terminus, to either the T18 or T25 domain of the Bordetella adenylate cyclase. A positive interaction was observed by the appearance of blue color. Experiments were repeated at least 3 times, and representative photographs are shown. In the plates, four serial dilutions of transformed cells were spotted. (D) DisA binds RuvA-HJ DNA complexes. [ $\left.\gamma-{ }^{32} \mathrm{P}\right] \mathrm{HJ}$ DNA was incubated with the indicated RuvA and/or DisA concentrations for 15 min in buffer C containing $1 \mathrm{mM} \mathrm{MgCl}_{2}$ at $37^{\circ} \mathrm{C}$. The protein-HJ DNA complexes were separated by $6 \%$ native 
PAGE. -, no protein added. RuvA bound to HJ forms two types of complex (R-I and R-II), DisA$\mathrm{HJ}$ mainly one type of complex (A) and RuvA-HJ-DisA (R+A) complexes. (E) DNase I footprint analyses. $\left[\gamma_{-}{ }^{32} \mathrm{P}\right] \mathrm{HJ}$ DNA was pre-incubated with a fixed amount of RuvB $(80 \mathrm{nM})$, and increasing concentrations of RuvA ( 40 and $80 \mathrm{nM}$ ) or DisA ( 60 and $120 \mathrm{nM}$ ) in buffer C containing $5 \mathrm{mM}$ ATP $\gamma \mathrm{S}$ and $10 \mathrm{mM} \mathrm{MgCl}_{2}$ (for $15 \mathrm{~min}$ at $37^{\circ} \mathrm{C}$ ). Then, the second protein (DisA or RuvAB) was added, and the reaction incubated $\left(15 \mathrm{~min}\right.$ at $\left.37^{\circ} \mathrm{C}\right)$. Finally, DNAse I was added. C, the HJ DNA control without DNase I, and in lanes 1 and 17 with DNase I treatment. The regions protected by the individual proteins or by both proteins are marked with rectangles. The order of protein addition is indicated at the top. The position of the ssDNA crossover is indicated as 'junction'. The hypersensitive sites characteristic of RuvA binding are highlighted by an asterisk. Experiments were repeated at least 3 times, and a representative gel is shown. (F) PFGE of $w t$ and $\Delta$ dis $A$ cells after treatment or not with $10 \mathrm{mM}$ MMS for 20 min before the preparation of plugs. *, DSB compression zone (above $600 \mathrm{~kb}$ ), smaller DSBs are detected as a smear. Markers used are Lambda PFG ladder (M1) and Lambda DNA HindIII digest (M2). (G) Quantification of the chromosomal fragmentation. The increase in DSBs was plotted relative to the $w t$ untreated condition, which is given a value of 1 . Results are the mean plus the SD of three independent experiments.

The coding sequence of the genes was fused to the $5^{\prime}$ or $3^{\prime}$ sequence of either the T18 or T25 region of the Bordetella adenylate cyclase gene, as described [18]. Using this system, we confirmed that DisA, RuvA, or RuvB interact with themselves (Figure 2A-C), because such interaction activates the $\mathrm{CAMP}$-bound catabolite activator protein to induce the expression of $\beta$-galactosidase. This leads to the appearance of blue-colored colonies, due to the breakdown of X-gal in the medium, as observed for the Zip control (Figure 2A-C). We observed that the interaction of DisA with RuvB induces $\beta$-galactosidase expression (Figure 2A), whereas a physical interaction of DisA with RuvA was not deduced (Figure 2C).

The DisA HhH domains structurally resemble that of RuvA [22]. To evaluate whether DisA interacts with RuvB through its C-terminal HhH RuvA-like DNA-binding domain, the dis $A \triangle \mathrm{C} 290$ mutant gene [18] was fused to the T18 or T25 regions. The interaction of RuvB with DisA $\Delta$ C290 variant induced $\beta$-galactosidase expression to levels comparable to that of the Zip control (Figure 2B). It is likely that the DNA-binding domain of DisA is dispensable for its interaction with RuvB.

\subsection{DisA Coexists with RuvAB on HJ DNA}

To test whether DisA works in concert with RuvAB, the RuvA and RuvB proteins were purified and EMSAs were performed. The RuvB-DisA interaction could not be studied

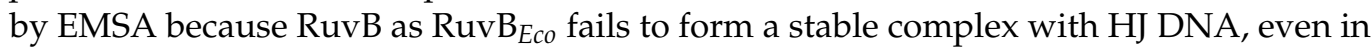
the presence of the non-hydrolysable ATP analogue ATP $\gamma S$ and of $0.2 \%$ glutaraldehyde addition to fix any pre-existing RuvB-DNA complex (data not shown). Similar results were previously reported $[37,41]$. RuvAB forms a slow-moving complex with HJ DNA that is trapped in the well [41]. Therefore, the existence of a hypothetical DisA-HJ DNA-RuvAB complex could not be distinguished from the DisA-HJ DNA complex using EMSA (see Figure 1B). The first step in $\mathrm{HJ}$ resolution by the RuvAB-RecU resolvasome is the formation of the RuvA-HJ DNA complex [41]. Thus, the formation of putative RuvA-HJ DNA-DisA complexes was analyzed by EMSA.

The RuvA protein binds HJ-J3 DNA preferentially in the presence of EDTA and binding is strongly reduced in the presence of $10 \mathrm{mM} \mathrm{MgCl}_{2}$ [49-51]; therefore, the experiments were performed in the presence of $1 \mathrm{mM} \mathrm{MgCl}_{2}$, although this is not the optimal condition for DisA binding (see Supplementary Materials and Figure S1A,B). RuvA bound $\left[\gamma^{32} \mathrm{P}\right]$ HJ-J3 DNA with a $K_{\text {Dapp }}$ of $10 \pm 2 \mathrm{nM}$, and saturating RuvA concentrations led to the formation of a slow-moving R-II complex (Figure 2D, lanes 2 and 3), which corresponds to two RuvA tetramers bound to the same HJ molecule, as observed previously in the presence of EDTA [50]. DisA bound [ $\left.\gamma^{32} \mathrm{P}\right]-\mathrm{HJ}-J 3$ DNA and formed large molecular mass complexes that mainly remained trapped in the well (Figure 2D, lane 5), as the ones observed at high $\mathrm{Mg}^{2+}$ concentrations (Figure 1B). DisA seemed to facilitate RuvA-HJ DNA 
complex formation (Figure 2D, lane 6 vs. 2), and a new RuvA-HJ-J3 DNA-DisA complex $(\mathrm{R}+\mathrm{A})$ was observed (Figure 2D, lanes 7 and 9). It is likely that DisA and RuvA bound HJ DNA with a certain degree of cooperativity, and that both proteins may co-exist in the same HJ DNA molecule.

To address whether DisA and RuvAB co-localize and interact with the HJ, a DNase I footprint assay was performed. The protein- $\left[\gamma^{32} \mathrm{P}\right]-\mathrm{HJ}-\mathrm{J} 3 \mathrm{DNA}$ complexes were pre-formed ( $5 \mathrm{~min}$ at $37^{\circ} \mathrm{C}$ ), and then fixed DNase I, $5 \mathrm{mM} \mathrm{ATP} \gamma \mathrm{S}$ and $\mathrm{MgCl}_{2}$ up to $10 \mathrm{mM}$ were added, because DNase I requires $10 \mathrm{mM} \mathrm{MgCl}_{2}$ for its catalytic activity (Figure 2E). The junction or cross-over region of the HJ DNA is single stranded, and thus insensitive to DNase I attack (Figure 2E, junction). RuvB.ATP $\gamma$ S did not protect the HJ DNA from DNase I attack, confirming that RuvB needs to be recruited on the HJ DNA (Figure 2E, lane 2). RuvA, bound at the junction, protected both arms of the labeled $\mathrm{HJ}$ strand, with a footprint of $\sim 26-\mathrm{nt}$, and revealed some hypersensitive sites (Figure 2E, lanes 3 and 4, frame and asterisks). RuvA even protected the DNA substrate from the observed spontaneous cleavage (see lane $\mathrm{C}$, control). In the presence of stoichiometric RuvB.ATP $\gamma \mathrm{S}$ and RuvA, HJ-J3 DNA was protected from DNase I attack, with an extended footprint of $\sim 40$-nt (Figure 2E, framed lanes 5 and 6). This extended footprint was also observed for the RuvAB ${ }_{E c o}$ complex [52].

DisA binding showed an extended region of protection from DNase I attack (40-45 bp DNA) (Figure 2E, lanes 7 and 8) [27]. This is consistent with the hypothesis that the DisA $\mathrm{HhH}$ domains interact with the different arms of the HJ, forming large complexes as observed above. These results indirectly suggested that DisA could prevent degradation of the extruded nascent strands of a reversed fork. At present, we cannot rule out that more than one DisA octamer is recruited to the HJ DNA.

When DisA was added to pre-formed RuvAB-HJ DNA complexes or vice versa, at a DisA:RuvAB molar ratio of 1.5:1 to 3:1, an extended region of protection from DNase I attack, similar to those for DisA, was observed (Figure 2E, lanes 5-8 vs. 9-16). The footprint also showed the patterns observed for RuvAB, as the specific hypersensitive bands (denoted by asterisks), suggesting its presence on the HJ DNA together with DisA. Since the stoichiometry of the observed signals is similar in the different tracks, we are confident that both DisA and RuvAB are bound to the same molecules of HJ DNA.

\subsection{Dis A May Protect Cells from Chromosome Breaks}

DNase I footprinting studies suggested that DisA might shield branched intermediates so that it could have protecting activity in vivo, thus avoiding the degradation of the reversed fork. We then tested whether DisA protects DNA from degradation during rapid growth in LB medium in unperturbed conditions or upon MMS-induced replicative stress, quantifying the accumulation of chromosomal fragments in $w t$ and $\triangle$ dis $A$ cells by pulsed-field gel electrophoresis (PFGE) (Figure 2F). It is worth noting that circular DNA and chromosome-sized branched replication intermediates are unable to migrate into pulsed-field gels and remain in the well [20,53].

In the absence of DNA damage, a modest but significative increase in the amount of degraded DNA was observed in $\triangle$ dis $A$ cells when compared to the wt control $(p<0.01)$ (Figure 2F,G). To test whether this degradation is due to RuvAB-RecU-mediated cleavage of the reversed fork as observed in E. coli [10] or during canonical DSB repair [37], we tested chromosomal degradation in the $\Delta r e c U$ context (Figure S3). In the absence of the RecU HJ resolvase, no significative decrease in the amount of degraded DNA was observed $(p>0.5)$ (Figure S3). It is likely that in the absence of DisA, prolonged replication stress may cause replication fork collapse and degradation but not in the absence of RecU.

Then, DNA replication was perturbed by the addition of MMS. MMS, which mainly methylates purines, causes replication fork slowing and fork stalling [20]. In the presence of $10 \mathrm{mM}$ MMS, which reduces $\sim 3$-fold the survival of $w t$ cells [23], an increase in DNA fragmentation was observed when compared to the absence of MMS $(p<0.05)$ (Figure 2F). The quantitative analysis of three independent experiments also revealed the increase of chromosomal fragmentation in $\triangle$ dis $A$ cells upon treatment with MMS. The levels of chro- 
mosomal degradation in the $\Delta$ dis $A$ context were higher than in the $w t$ control (Figure $2 \mathrm{G}$ ). These results suggest that: (i) DisA may contribute to protect paused or stalled forks from degradation in unperturbed replicating cells, and (ii) MMS induces chromosomal fragmentation in both wt and $\Delta$ dis $A$ cells.

\subsection{DisA Cannot Activate RuvAB to Catalyze Fork Reversal}

In E. coli, $\mathrm{HJ}$ intermediates accumulate in $\triangle r u v A B$ cells but not in the $\triangle r u v A B \triangle r e c G$ context [54], suggesting that Ruv $A_{E c o}$ by itself may not reverse a stalled fork. To test whether RuvAB converts a stalled fork with a gap in the leading or in the lagging strand onto a reversed fork, and if DisA affects the rate of such conversion, two synthetic stalled replication forks, with a 15-nt ssDNA gap either on the leading strand (forked-Lead) or on the lagging strand (forked-Lag), were used. These substrates have heterologous arms to prevent spontaneous branch migration; thus, if RuvAB mediates fork reversal, the resulting product should migrate as a flayed DNA (unreplicated fork) (Figure 3).

A

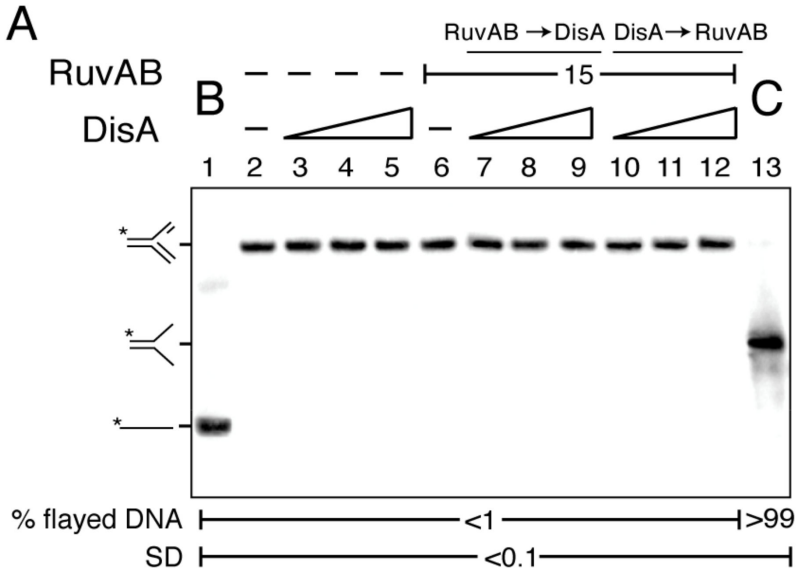

C

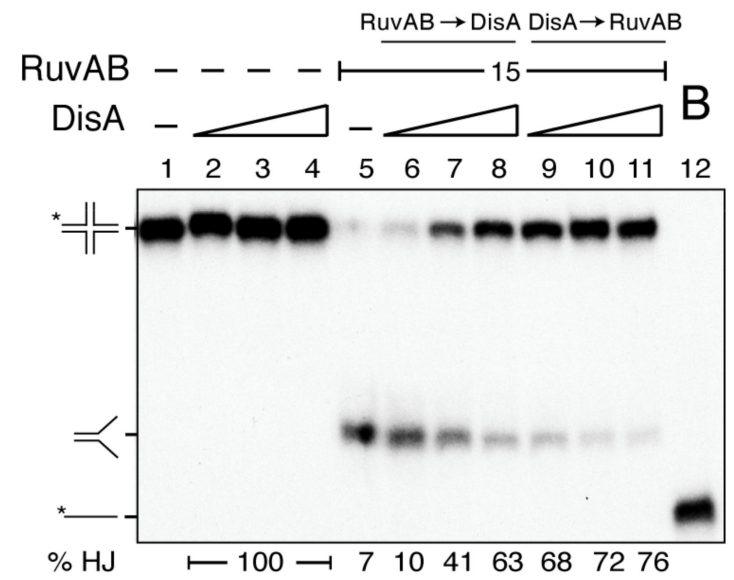

B
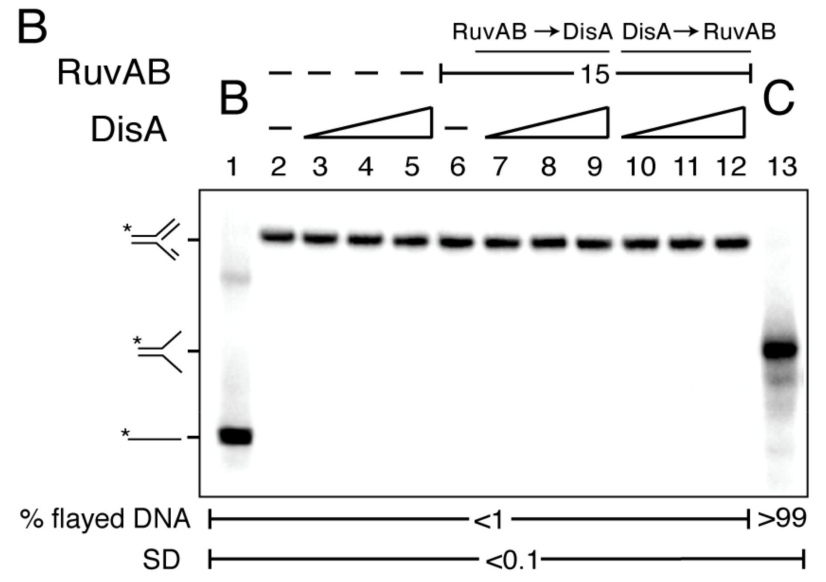

$\mathrm{D}$

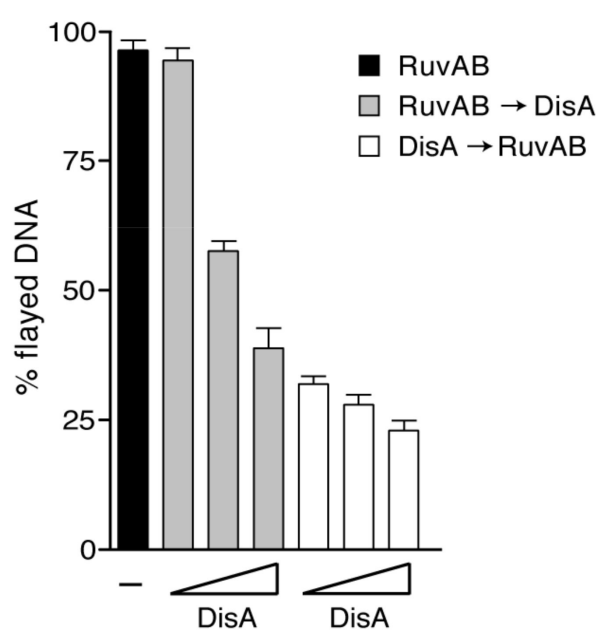

Figure 3. DisA action on RuvAB translocase activity. $(\mathbf{A}, \mathbf{B})$ RuvAB fails to convert a gapped stalled fork into a reversed one. $\left[\gamma_{-}{ }^{32} \mathrm{P}\right]$ forked-Lead $(\mathbf{A})$ or $\left[\gamma^{-}{ }^{32} \mathrm{P}\right]$ forked-Lag DNA (B) was pre-incubated with increasing DisA concentrations (doubling from 12-48 nM) or a fixed amount of RuvAB $(15 \mathrm{nM})$ in buffer $C$ containing $10 \mathrm{mM} \mathrm{MgCl} 2\left(15 \mathrm{~min}\right.$ at $\left.37^{\circ} \mathrm{C}\right)$. Then, the second protein (variable DisA [RuvAB $\rightarrow$ DisA] or a constant amount of RuvAB [DisA $\rightarrow$ RuvAB]) and $5 \mathrm{mM}$ ATP were added, and the reaction was further incubated $\left(15 \mathrm{~min}\right.$ at $\left.37^{\circ} \mathrm{C}\right)$. The reaction was stopped, deproteinized, and separated by $6 \%$ native PAGE. -, no protein added; B, boiled forked substrate (lane 1); ${ }^{*}$, radiolabelled strand; C, expected products of the helicase activity loaded as running position controls. Representative gels are shown. (C) DisA inhibits RuvAB-mediated fork restoration. $\left[\gamma^{32}{ }^{32} \mathrm{P}\right] \mathrm{HJ}-\mathrm{J} 4 \mathrm{DNA}$ was pre-incubated with increasing DisA concentrations or a fixed amount of RuvAB in buffer $\mathrm{C}$ containing $10 \mathrm{mM} \mathrm{MgCl} 2\left(15 \mathrm{~min}\right.$ at $\left.37^{\circ} \mathrm{C}\right)$. Then, the second protein (variable DisA [RuvAB $\rightarrow$ DisA] or a constant amount of RuvAB [DisA $\rightarrow$ RuvAB]) and $5 \mathrm{mM} \mathrm{ATP}$ were added, and the reaction was further incubated $\left(15 \mathrm{~min}\right.$ at $\left.37^{\circ} \mathrm{C}\right)$. 
The reaction was stopped, deproteinized, and separated by $6 \%$ native PAGE. -, no protein added; B, boiled substrate. The percentage of remaining substrate $(\mathrm{HJ})$ is indicated at the bottom. (D) The relative amount of flayed DNA produced in three independent experiments as the one shown in $(C)$ was quantified. Values are represented as the mean \pm SEM.

In the presence of ATP, RuvAB ( $15 \mathrm{nM})$ did not unwind forked-Lead or forked-Lag DNA, and a flayed intermediate that would be the product of the backwards pushing of the replicated fork to displace both nascent strands was not observed (Figure 3A,B, lane 6). In contrast, when the branch migrating translocase RecG (15 nM) was incubated with these forked-Lead or forked-Lag DNA substrates and ATP, the flayed DNA product was observed [27].

To test whether DisA changes this outcome by its interaction with RuvB, increasing DisA concentrations were added to the reactions with the forked-Lead or forked-Lag DNA substrates. As expected, DisA does not show any activity over these substrates (Figure 3A,B, lanes 3-5). Independently of the order of protein addition, DisA did not facilitate RuvAB-mediated formation of reversed forks by branch migrating these DNA structures (Figure $3 A, B$, lanes 7-12 vs. 6). Similarly, stalled forks are poor substrates for $\operatorname{RuvAB}_{E c o}$ in vitro [31].

\subsection{DisA Bound to HJ DNA Inhibits RuvAB-Mediated Fork Restoration}

In vitro, both RuvAB $B_{E c o}$ and RuvAB efficiently branch migrate an HJ-like structure to restore a replication fork $[30,31,41]$. We showed that in the presence of ATP, RuvAB $(15 \mathrm{nM})$, bound to a HJ (HJ-J4 DNA), produced the flayed duplex product, but it failed to further unwind the flayed duplex intermediate to render an ssDNA substrate (Figure 3C, lane 5 vs. 12). Similar results were observed when RuvAB was incubated with HJ DNA that cannot spontaneously branch migrate (HJ-J3 DNA) [41]. DisA did not unwind the HJ-J4 DNA (Figure 3C, lanes 2-4).

We tested whether DisA affects RuvAB-mediated fork restoration. DisA pre-incubated with HJ-J4 DNA significantly inhibited RuvAB-mediated branch migration $(p<0.01)$ even at a DisA:RuvAB molar ratio of 0.8:1 (Figure 3C, lane 9 and 3D). When DisA was added to the pre-formed RuvAB-HJ-J4 DNA complexes, however, no inhibition was observed at a DisA:RuvAB molar ratio of $0.8: 1$, but at a 1.6:1 DisA:RuvAB molar ratio, 50\% of the HJ-J4 DNA was not unwound (Figure 3C, lanes 6 and 7 and 3D). In the presence of an excess of DisA (DisA:RuvAB molar ratio of 3:1), RuvAB-mediated branch migration was significantly inhibited $(p<0.01)$ (Figure 3C, lane 11 and 3D). The different outcome observed by altering the order of protein addition reveals a genuine activity associated with DisA rather than any inhibitory component present in the protein preparation (e.g., a protease). We envision that DisA bound to the HJ DNA blocks branch migration. Alternatively, DisA acting as a potent roadblock inhibits any translocase, including RuvAB. Nevertheless, we consider this hypothesis unlikely, because the replicative accessory DNA helicase PcrA (a member of the conserved UvrD-FBH1 family of $3^{\prime} \rightarrow 5^{\prime}$ DNA helicases) efficiently processes a tailed HJ-DNA even in the presence of a 6-fold excess of DisA relative to PcrA [27]. Furthermore, DisA neither compromises PriA-dependent replication initiation nor affects replication elongation using an in vitro reconstituted replication assay [13]. These results confirm that the inhibitory action exerted by DisA is specific for RuvAB.

\subsection{DisA Suppresses the DNA-Dependent ATPase Activity of RuvAB}

To understand the molecular basis of the inhibition of the RuvAB motor, we tested whether DisA inhibits RuvAB-mediated hydrolysis of ATP. As previously reported for $\operatorname{RuvA}_{E c o}$ or RuvA [37,41,55], RuvA lacked any ATPase activity $\left(\mathrm{K}_{\text {cat }}\right.$ of $\left.<0.1 \mathrm{~min}^{-1}\right)$ in the presence or absence of ssDNA (Table 1). In the absence of DNA, RuvB or the RuvAB complex $(15 \mathrm{nM})$ hydrolyzed ATP with a similar $\mathrm{K}_{\text {cat }}\left(\sim 280 \pm 7 \mathrm{~min}^{-1}\right)$ (Table 1). Similarly, $\operatorname{RuvB}_{E c o}$ can hydrolyze ATP in the absence of any DNA [37,55]. The DAC activity of DisA does not form ADP, the product that our assay is measuring [18]. 
Table 1. RuvAB rates of ATP hydrolysis.

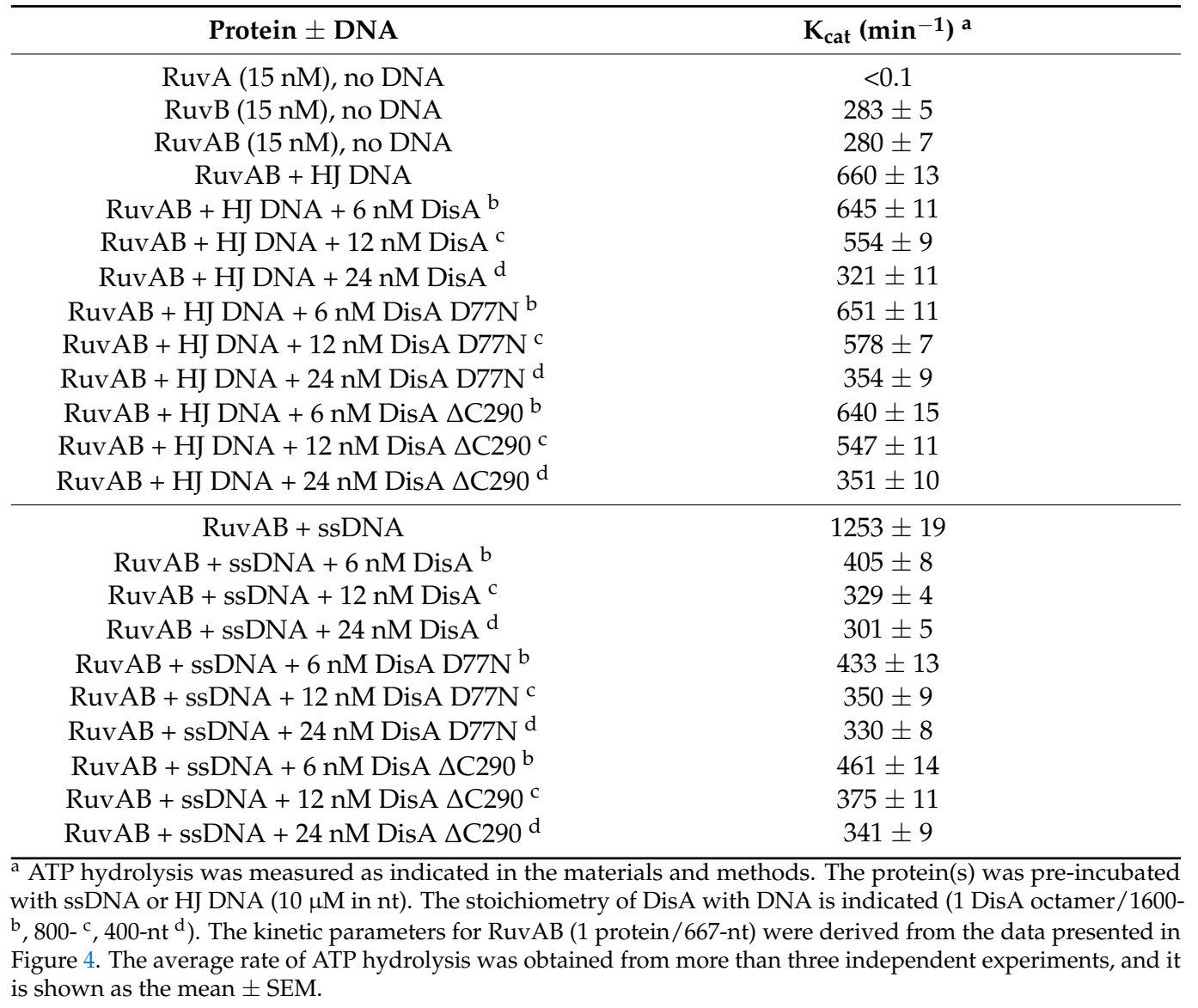

In the presence of circular pGEM-3Zf $(+)$ ssDNA (cssDNA, $10 \mu \mathrm{M}$ in nt), the ATPase activity of RuvAB (1 RuvAB complex/666-nt) was stimulated by $\sim 5$-fold $(p<0.01)$, but it only increased $\sim 2$-fold $(p<0.05)$ in the presence of HJ-J3 DNA (10 $\mu \mathrm{M}$ in nt) (Figure $4 \mathrm{~A}$ vs. Figure $4 \mathrm{~B}$, dark blue line, Table 1), as observed for RuvAB $\mathrm{B}_{E c o}$ [31]. In the presence of $10 \mathrm{mM}$ $\mathrm{Mg}^{2+}$, cssDNA adopts secondary structures with single- (mimicking an unreplicated fork) and double-hairpin motifs (analogous to a HJ). It is likely that these structures mimic the preferred structures for RuvAB binding (Figure $4 \mathrm{~A}$ vs. Figure $4 \mathrm{~B}$ ), as earlier reported for $\operatorname{RuvAB}_{E c o}[56]$.

In the presence of cssDNA and limiting DisA concentrations $(6 \mathrm{nM}, \sim 2$ DisAs/cssDNA molecule), RuvAB-mediated ATP hydrolysis was significantly reduced (by $\sim 4$-fold, $p<0.01$ ) (Figure 4A, orange line, Table 1). Moreover, in the presence of nearly stoichiometric DisA concentrations (12 nM) with respect to RuvAB, the maximal rate of ATP hydrolysis was comparable to that of RuvAB in the absence of DNA ( $p>0.1$ ) (Figure $4 \mathrm{~A}$, green vs. dark blue line, Table 1). Similarly, in the presence of HJ-J3 DNA and DisA, the rate of RuvABmediated ATP hydrolysis was comparable to that of RuvAB in the absence of DNA ( $p>0.1)$ (Figure 4B, brown vs. dark blue line, Table 1). It is likely that DisA interacts with and inhibits the ssDNA-dependent activity of RuvB (Figure 2A,B), but it does not affect the DNA-independent ATPase activity of RuvB (or of the RuvAB complex). 

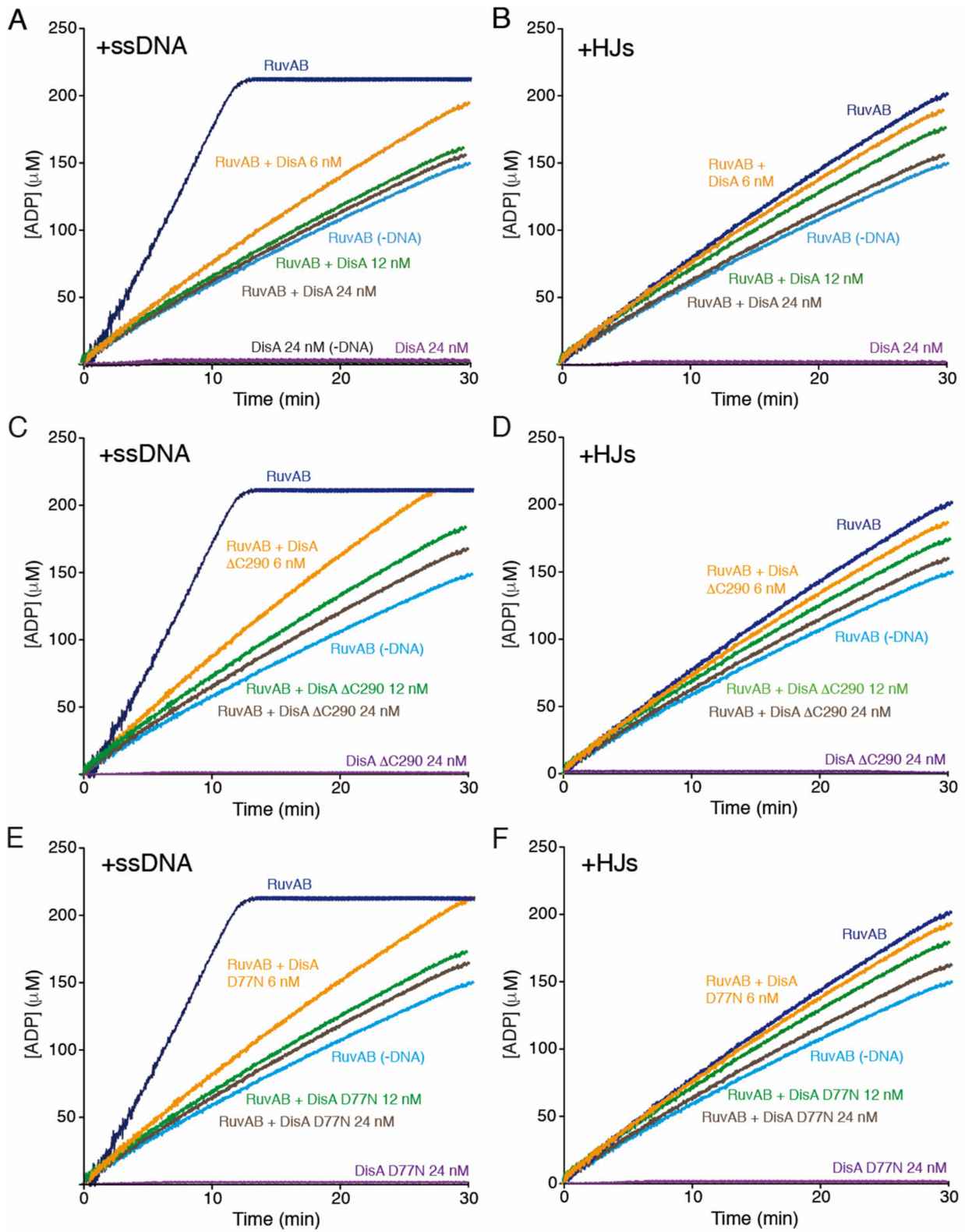

Figure 4. DisA inhibits RuvAB-mediated ATP hydrolysis. (A,B) The ATPase activity of the RuvAB complex (15 nM) in buffer B containing $5 \mathrm{mM}$ ATP was measured during 30 min with circular 3199-nt ssDNA (in A) or HJ DNA (in B) as the effector. As indicated, DisA (6, 12 and $24 \mathrm{nM}$ ) was added, or DNA was omitted. (C-F) DisA inhibits RuvAB-mediated ATP hydrolysis by a direct interaction. Circular 3199-nt ssDNA (10 $\mu$ M in nt) (C,E) or HJ DNA (D,F) was incubated with RuvAB $(15 \mathrm{nM})$ and DisA $\triangle \mathrm{C} 290(\mathbf{C}, \mathbf{D})$ or DisA D77N (E,F) (6 to $24 \mathrm{nM})$ in buffer B containing $5 \mathrm{mM}$ ATP, and the ATPase activity was measured $\left(30 \mathrm{~min}\right.$ at $\left.37^{\circ} \mathrm{C}\right)$. All reactions were repeated three or more times with similar results, representative graphs are shown here, and the mean $\mathrm{K}_{\mathrm{cat}} \pm \mathrm{SEM}$ are listed in Table 1.

\subsection{DisA Does Not Compete with RuvAB for the Binding to DNA}

DisA might compete with RuvAB for DNA binding and inhibit its ssDNA-dependent ATPase activity. To test this hypothesis, DisA was replaced by DisA $\triangle$ C290. This mutant, which lacks the DNA-binding domain but retains its DAC activity [24], interacts with RuvB (Figure 2B). In the presence of ssDNA or HJ DNA, RuvAB-mediated ATP hydrolysis was inhibited by DisA $\Delta$ C290, to levels comparable to $w t$ DisA (Figure $4 A$, B vs. Figure 4 C,D, orange, green, and brown vs. dark blue line, Table 1). This suggests that DisA does not inhibit RuvAB-mediated ATPase activity due to competition for DNA binding. Since DisA neither inhibits the DNA-independent RuvB (or RuvAB) ATPase nor competes with RuvAB 
for binding to its DNA substrate, we assumed that the inhibition of the RuvAB ATPase by DisA is a genuine and specific activity of the latter. Furthermore, DisA does not affect the ATPase activity of the unrelated PcrA DNA helicase [27].

\subsection{The DAC Activity of DisA Does Not Compromise RuvAB-Mediated ATP Hydrolysis}

DisA might exhaust the ATP pool with its DAC activity, or the c-di-AMP produced might poison RuvAB ATPase activity. To examine this hypothesis, DisA was replaced by the DisA D77N variant, which cannot synthesize c-di-AMP $[18,22]$. In the presence of ssDNA or HJ-J3 DNA and DisA D77N, RuvAB-mediated ATP hydrolysis was inhibited to a comparable level to that when $w t$ DisA was used (Figure 4A,B vs. Figure 4E,F, orange, green, and brown vs. dark blue line, Table 1). Therefore, neither the DAC activity of DisA with subsequent consumption of ATP, nor c-di-AMP, are involved in the reduction of RuvAB-mediated ATP hydrolysis. Altogether, these data suggest that DisA inhibits the ATPase activity of the RuvAB-ssDNA complex by its direct interaction with RuvB.

\subsection{DisA Does Not Interact with RecU}

With few exceptions, the RecU HJ resolvase constitutes, together with the RuvAB complex, the resolvasome in bacteria of the Firmicutes and Tenericutes phyla [41]. Genetic studies suggest that $\operatorname{dis} A$ is epistatic to the recA or recU gene in response to DNA damage $[13,23]$. The RecA protein physically interacts with both DisA and RecU $[18,57]$. Upon cell envelope stress, the expression of both the dis $A$ and the recU genes is significantly increased [35,36], suggesting a temporal and functional link between them. Therefore, a functional interaction of DisA and RecU was explored. First, we analyzed whether RecU and DisA physically interact using the bacterial two-hybrid system. RecU interacts with itself, in agreement with the X-ray data that show that the protein is a dimer [42]. A stable interaction of RecU with DisA, however, was not observed (Figure 5A).

A

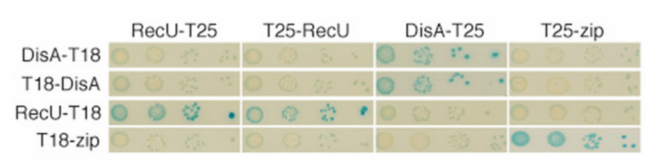

B

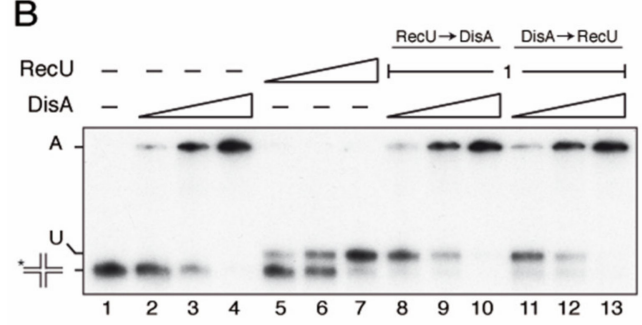

D

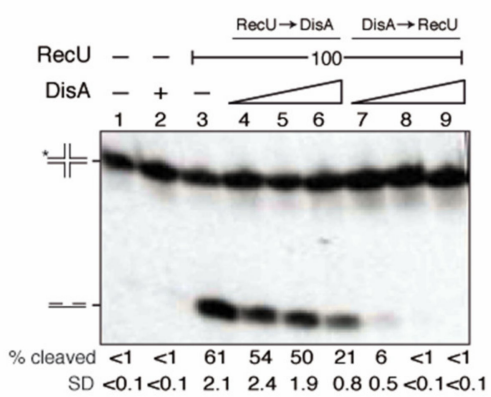

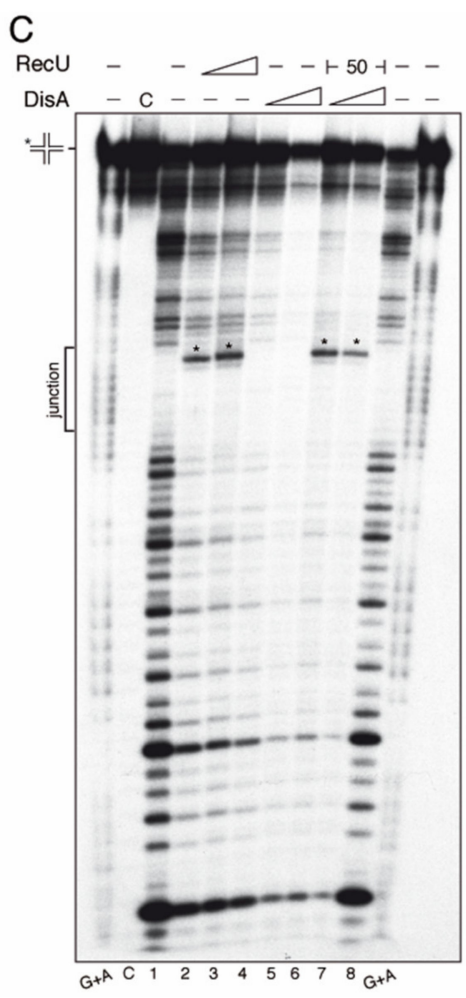

Figure 5. DisA does not interact with RecU but inhibits RecU-mediated HJ cleavage. (A) DisA interaction with RecU was not observed by the bacterial two-hybrid interaction assay. $(\mathbf{B})\left[\gamma^{-32} \mathrm{P}\right] \mathrm{HJ}$ DNA was pre-incubated with increasing DisA concentrations (doubling from 12-48 nM) or RecU (doubling from $0.25-1 \mathrm{nM}$ ) for $15 \mathrm{~min}$ in buffer $\mathrm{C}$ containing $1 \mathrm{mM} \mathrm{MgCl} 2\left(5 \mathrm{~min}\right.$ at $\left.37^{\circ} \mathrm{C}\right)$. Then, 
the second protein (variable DisA or a constant amount of RecU) was added and the reaction was incubated $\left(15 \mathrm{~min}\right.$ at $\left.37^{\circ} \mathrm{C}\right)$. The protein-HJ DNA complexes were separated by $6 \%$ native PAGE. -, no protein added. The RecU-HJ (U) and DisA-HJ (A) complexes are shown. The order of protein addition is indicated at the top. (C) Autoradiogram showing a footprint analysis of the binding of RecU and DisA to HJ DNA. $\left[\gamma^{-32} \mathrm{P}\right] \mathrm{HJ}$ DNA was pre-incubated with increasing concentrations of $\operatorname{RecU}(50$ and $100 \mathrm{nM})$ or DisA $(60$ to $120 \mathrm{nM})\left(5 \mathrm{~min}\right.$ at $\left.37^{\circ} \mathrm{C}\right)$ in buffer C containing $1 \mathrm{mM} \mathrm{MgCl}_{2}$. Then, the second protein ( $\operatorname{RecU} 50 \mathrm{nM}$ ) was added, and the reaction incubated $\left(15 \mathrm{~min}\right.$ at $\left.37^{\circ} \mathrm{C}\right)$. After that, DNase I and $\mathrm{MgCl}_{2}$ up to $10 \mathrm{mM}$ were added. C, no DNase I was added. The position of the ssDNA crossover is indicated as 'junction' and RecU-mediated cleavage of the [ $\left.\gamma^{-}{ }^{32} \mathrm{P}\right]$-labelled arm is indicated by an *. The G + A marker is indicated. Experiments were repeated at least 3 times, and a representative gel is shown. (D) $\left[\gamma^{-32} \mathrm{P}\right] \mathrm{HJ}$ DNA labelled on arm 1 (denoted by ${ }^{*}$ ) was pre-incubated with DisA (doubling from $24-96 \mathrm{nM})$ or $\operatorname{RecU}(100 \mathrm{nM})\left(5 \mathrm{~min}\right.$ at $\left.37^{\circ} \mathrm{C}\right)$ in buffer $\mathrm{C}$ containing $1 \mathrm{mM}$ $\mathrm{MgCl}_{2}$. Then, the second protein (variable DisA [RecU $\rightarrow$ DisA] or a constant amount of RecU [DisA $\rightarrow \operatorname{RecU}])$, and $\mathrm{MgCl}_{2}$ up to $10 \mathrm{mM}$ were added, and the reaction was incubated $\left(15 \mathrm{~min}\right.$ at $\left.37^{\circ} \mathrm{C}\right)$. The reaction was stopped, deproteinized, and analyzed by $15 \%$ denaturing PAGE, no protein added. The relative amount of cleaved DNA in three independent experiments was quantified as described, and a representative gel, the mean \% of cleaved DNA, and its SD are shown.

To analyze whether DisA modulates the mechanism of RecU-mediated HJ resolution, the RecU HJ resolvase was purified, and DNA binding was analyzed. In the presence of $1 \mathrm{mM} \mathrm{Mg}^{2+}$, RecU specifically binds HJ DNA, and in the presence of $10 \mathrm{mM} \mathrm{Mg}^{2+}$, RecU binds and cleaves the $\mathrm{HJ}$ structure when its cognate site is exposed $[42,46]$. Therefore, to perform DNA binding studies, we used $1 \mathrm{mM} \mathrm{MgCl} 2$, which it is not the optimal condition for DisA binding (see Supplementary Materials, and Figure S1).

RecU binds HJ-J3 DNA with high affinity ( $\mathrm{K}_{\text {Dapp }}$ of $0.6 \pm 0.2 \mathrm{nM}$; Figure $5 \mathrm{~B}$, lanes 5-7), as described [46] and with $\sim 30$-fold higher affinity than DisA under this experimental condition. When HJ-J3 DNA was incubated with a fixed amount of RecU and increasing DisA concentrations, independently of the order of addition, protein-HJ DNA complexes became entrapped in the well (Figure 5B, lanes 9, 10,12 and 13), so that the presence of a putative RecU-HJ-DisA complex remained poorly defined.

To further analyze a potential interaction, we made DNAse I protection footprint assays. Since DNase I requires $10 \mathrm{mM} \mathrm{MgCl} 2$ for its activity, but under this condition RecU binds and cleaves the $\mathrm{HJ}$ structure, the $\left[\gamma^{32} \mathrm{P}\right]-\mathrm{HJ}-\mathrm{J} 3 \mathrm{DNA}$-protein complexes were first pre-formed at $1 \mathrm{mM} \mathrm{Mg}{ }^{2+}\left(5 \mathrm{~min}\right.$ at $37^{\circ} \mathrm{C}$ ). Then, fixed DNase I and $\mathrm{MgCl}_{2}$ up to $10 \mathrm{mM}$ were added (Figure 5C). RecU mainly interacts with the junction region, which is DNAse I resistant. Thus, a clear protected area was not observed. However, a defined band within the ssDNA junction was observed (Figure 5C, lanes 2 and 3, marked with an asterisk), whose position correlates with the expected cleavage of HJ DNA by RecU [46]. The footprint results are consistent with the observation that the RecU stalk region, by penetrating in the center of the $\mathrm{HJ}$, distorts it so that the $\mathrm{HJ}$ adopts a square planar conformation with a central hole [36,48]. In the presence of fixed RecU and increasing DisA concentrations, the band corresponding to RecU-mediated cleavage of HJ DNA significantly decreased, and the extended footprint of DisA was observed (Figure 5C, lanes 6 and 7). It is likely that both proteins co-exist on HJ DNA, and that DisA either alters the positioning of $\operatorname{RecU}$, the conformation of the HJ DNA, or both, thus affecting RecU-mediated HJ cleavage.

\subsection{DisA Limits RecU-Mediated HJ Cleavage}

The effect of DisA on RecU-mediated HJ cleavage was then analyzed. A large excess of DisA did not catalyze HJ cleavage (Figure 5D, lane 2). In the presence of $10 \mathrm{mM} \mathrm{Mg}^{2+}$, RecU cleaved the HJ-J3 to yield two nicked duplex products (Figure 5D, lane 3) $[46,48]$. When RecU was pre-incubated with HJ-J3 DNA ( 5 min $37^{\circ} \mathrm{C}$ ), the addition of a stoichiometric concentration of apo DisA only marginally affected RecU-mediated HJ-J3 cleavage ( $p>0.1)$ (Figure 5D, lane 3 vs. 6). In sharp contrast, when apo DisA was pre-incubated with HJ-J3 DNA, and then RecU was added, HJ cleavage was strongly inhibited even at a molar 
DisA:RecU ratio of 0.2:1 (Figure 5D, lane 7), and it was abolished in the presence of substochiometric DisA concentrations (Figure 5D, lanes 8 and 9). Since the inhibition exerted by DisA was dependent on the order of addition, we assumed that it was genuine. Here, ATP was not present in the reaction mixture, thus DisA-mediated c-di-AMP synthesis plays no role.

\subsection{RuvAB- or RecU-Bound HJ-J3 DNA Blocks DisA-Mediated C-Di-AMP Synthesis}

DisA catalyzes the synthesis of the essential c-di-AMP second messenger that regulates a wide variety of physiological functions and plays a central role in virulence in bacteria of the Firmicutes phylum [16]. DisA inhibits RuvAB- and RecU-mediated HJ processing (Figures 3D and 5D), and in the presence of HJ DNA, DisA-mediated c-di-AMP synthesis is inhibited [22,23]. We tested whether RuvAB or RecU regulates the DisA DAC activity at limiting ATP concentrations $\left(\mathrm{K}_{\mathrm{m}} 151 \pm 1.4 \mu \mathrm{M}\right)$ [27] to reduce the branch migrating activity of RuvAB that would disassemble the HJ DNA. Under the conditions used to detect radiolabeled $\left[\alpha_{-}{ }^{32} \mathrm{P}\right]-\mathrm{c}-\mathrm{di}-\mathrm{AMP},\left[\alpha^{-32} \mathrm{P}\right]-\mathrm{ADP}$ is poorly separated from the $\left[\alpha_{-}{ }^{32} \mathrm{P}\right]-$ ATP substrate; therefore, RuvAB-mediated ATP hydrolysis cannot be detected in our assay. In the presence of $100 \mu \mathrm{M}$ ATP and $10 \mathrm{mM} \mathrm{MgCl}_{2}$, DisA converted $70-80 \%$ of the ATP substrate into product (c-di-AMP), and traces of the pppA-pA intermediate were detected (Figure $6 \mathrm{~A}, \mathrm{~B}$, lane 2), as described [22,23]. The addition of RuvAB or RecU (30 nM) marginally affected the DAC activity of DisA ( $p>0.1$ ) (Figure $6 \mathrm{~A}, \mathrm{~B}$, lane 7 vs. 2).
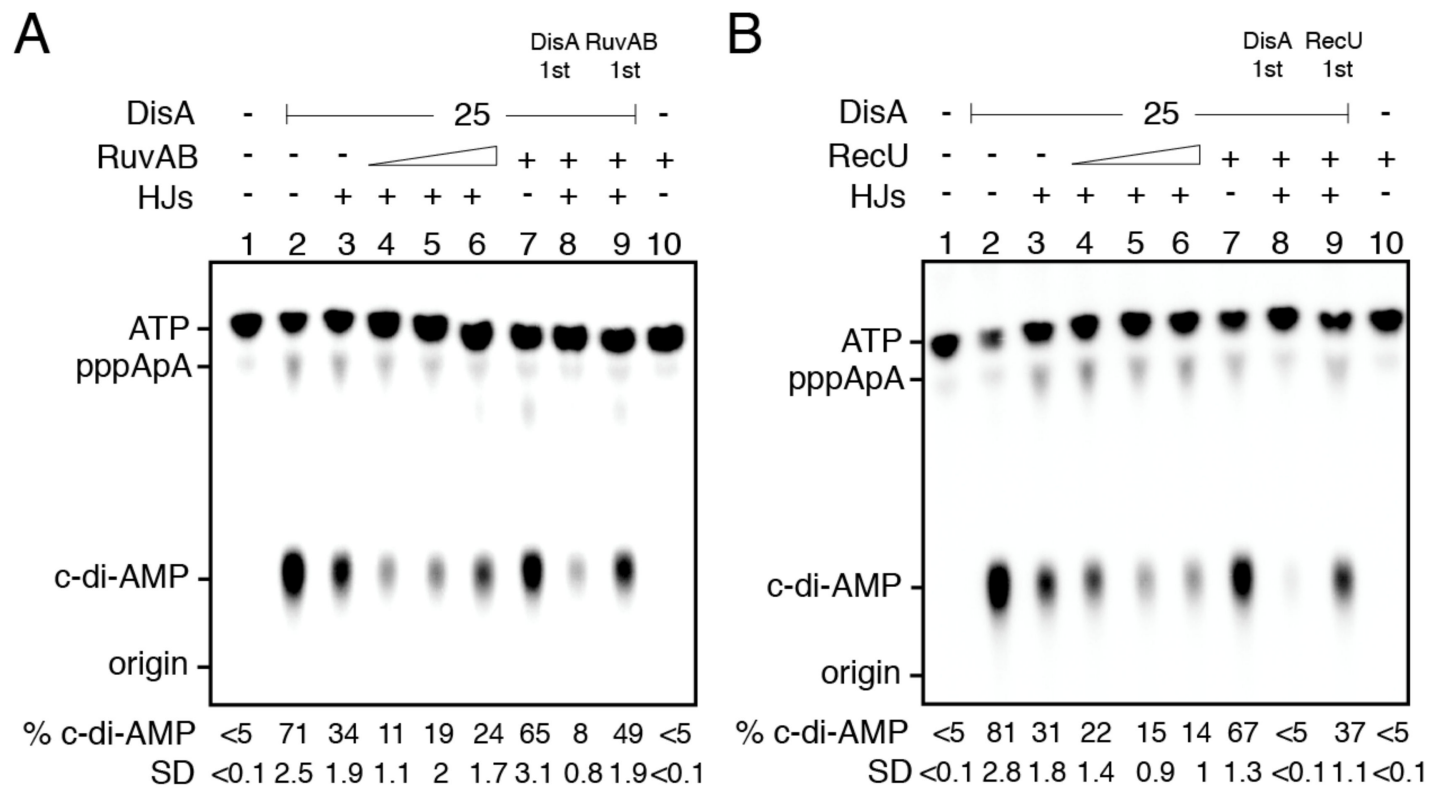

Figure 6. HJ DNA and RuvAB or RecU inhibits DisA-mediated c-di-AMP synthesis. (A,B) A fixed DisA concentration was incubated alone (lane 2), with HJ DNA (125 nM) (lane 3), increasing RuvAB (A) or RecU (B) concentrations (7, 15, or 30 nM) and HJ DNA (lanes 4-6), or with RuvAB (A) or RecU (B) (30 nM, lane 7) in buffer D containing $100 \mu \mathrm{M}$ ATP (at a ratio of 1:2000 [ $\left.\alpha^{32} \mathrm{P}\right]$-ATP:ATP) and $10 \mathrm{mM} \mathrm{MgCl}_{2}\left(30 \mathrm{~min}\right.$ at $\left.37^{\circ} \mathrm{C}\right)$. In lane 8, DisA was pre-incubated with HJ DNA (5 min at $37^{\circ} \mathrm{C}$ ) and then a fixed concentration of $\operatorname{RuvAB}(\mathbf{A})$ or $\operatorname{RecU}(\mathbf{B})$ and ATP were added, and the reaction incubated (30 min at $37^{\circ} \mathrm{C}$ ). In lane $9, \operatorname{RuvAB}$ or RecU was pre-incubated with HJ DNA and then DisA and ATP were added, and the reaction was incubated $\left(30 \mathrm{~min}\right.$ at $\left.37^{\circ} \mathrm{C}\right)$. In lane 10, a control reaction with only RuvAB or RecU (30 nM) is shown. The substrates and products were separated by TLC, and the spots quantified. The position of ATP, linear pppApA, c-di-AMP, and the origin are indicated. At least three independent experiments were performed, a representative plate, and the mean \% of c-di-AMP produced and its SD are shown.

As expected [22,23], in the presence of a fixed HJ-J3 DNA concentration, DisAmediated c-di-AMP synthesis was reduced by $\sim 3$-fold $(p<0.05$ ) (Figure $6 \mathrm{~A}, \mathrm{~B}$, lanes 3 vs. 2 ). In the presence of HJ-J3 DNA and RuvAB or RecU, several outcomes can be expected when 
the DisA DAC activity is assayed. First, RuvAB (or RecU) may displace DisA by competing for binding or by unwinding or cleaving, respectively, the HJ DNA, leading to the recovery of the DAC activity of DisA. Second, RuvAB (or RecU) may stabilize or relocate DisA on the HJ-J3 structure, thus additively affecting DisA-mediated c-di-AMP synthesis. Third, DisA bound to HJ DNA may not interact with RuvB, and the DAC activity is inhibited as in the presence of only HJ DNA. We found that in the presence of HJ-J3 DNA and a limiting RuvAB concentration (RuvAB:DisA 0.3:1 molar ratio), the DAC activity of DisA was additively inhibited ( 6-fold) $(p<0.01)$, suggesting that RuvAB can stabilize or relocate DisA on HJ-J3 DNA (see above). At RuvAB:DisA ratios approaching stoichiometry (0.6:1 and 1.2:1), RuvAB slightly reversed this negative effect, but the DisA DAC activity was still inhibited when compared with HJ DNA alone (Figure 6A, lane 3 vs. 5 and 6). To understand this mechanism of inhibition, the order of protein addition was varied. When DisA was pre-incubated with HJ-J3 DNA, and then RuvAB at a 1.2:1 RuvAB:DisA molar ratio was added, DisA-mediated c-di-AMP synthesis was strongly inhibited, suggesting that RuvAB stabilizes or relocates DisA on the HJ-J3 structure and this further impedes the DAC activity. However, if RuvAB was pre-incubated with the HJ-J3 DNA, the DAC activity of DisA was partially recovered at about stoichiometric concentrations (RuvAB:DisA 1.2:1 molar ratio) (Figure 6A, lane 9 vs. 3), perhaps because RuvAB translocates the HJ-J3 DNA. This suggests that there is a complex interplay between the three components (RuvAB, DisA, and HJ-J3 DNA). RuvAB bound to HJ DNA unwinds HJ structures, unless DisA is prebound to the HJ, and, on the other hand, DisA bound to HJ DNA suppresses its c-di-AMP synthesis in the presence of RuvAB.

Then, RuvAB was replaced by RecU. Increasing RecU concentrations in concert with HJ-J3 DNA synergistically inhibited the DAC activity of DisA (Figure 6B, lanes 4-6 vs. 7). When DisA was pre-incubated with HJ-J3 DNA, and then RecU was added, DisA-mediated c-di-AMP synthesis was strongly inhibited, suggesting that RecU may not displace DisA from the HJ-J3 structure (Figure 6B, lane 8 vs. 3). However, if RecU was pre-incubated with the HJ-J3 DNA, the DAC activity of DisA was partially recovered (Figure 6B, lane 9 vs. 3). These results suggest that RuvAB or RecU may stabilize and relocate DisA on the HJJ3 DNA, but the RuvAB-HJ DNA or RecU-HJ DNA complexes may process the DNA substrate and indirectly reduce the inhibition exerted by HJ DNA.

\section{Discussion}

In response to a replication stress, a stalled fork can be remodeled, but the function(s) that process(es) a stalled fork and the molecular basis of its regulation are poorly characterized in bacteria other than those of the $\gamma$-Proteobacteria class. Genetic analysis showed that when the single genome of an inert mature B. subtilis spore is exposed to ionizing radiation and then the spores are revived under unperturbed conditions, RecA, RecG, RuvAB, RecU, and DisA are required for survival, but neither RecQ-like (RecS and RecQ) remodelers nor the end-resection functions (AddAB and RecJ) are involved in spore revival [12,13].

Cytological studies have shown that DisA scans the chromosome and pauses at RecA bound to lesion-containing gaps at stalled replication forks rather than at DSBs during sporulation $[17,18]$. Dynamic DisA also pauses in unperturbed exponentially growing $\Delta r e c U$ cells but not in the exponentially growing $w t$ cells [23]. In the presence of MMS- or the UV mimetic 4-nitroquinoline 1-oxide (4NQO)-induced lesions, RecA filamented on the ssDNA gaps triggers the DNA damage response and stops DisA scanning, perhaps loading it at stalled or reversed forks [18]. DisA bound to branched intermediates reduces c-di-AMP synthesis to levels comparable to that in the absence of DisA, in otherwise $w t$ cells [21]. Low c-di-AMP levels indirectly inhibit cell proliferation (see the introduction).

Taking these data into account, we hypothesized that DisA could be providing, together with fork remodelers, a mechanism to cope with replicative stress and could protect stalled forks. Previously, it has been shown that during replication stress, DisA limits the activities of RecA and RecG [18,27]. Here, we show that DisA interacts with RuvB and inhibits the DNA-dependent ATPase activity of the RuvAB complex (Figures 2A,B and 4). 
RuvAB fails to reverse a stalled fork at protein concentrations that efficiently regress a reversed fork, and DisA cannot stimulate the conversion of a stalled into a reversed fork (Figure 3). It is likely that during a replication stress, RecG (or RecA) converts a stalled fork into a reversed fork [27]. The reversed fork may be further processed by the RuvAB-RecU resolvasome to yield a one-ended DSB and a nicked duplex, as reported in E. coli $[10,28,29]$. However, if this occurs, these physiological reversed forks intermediates would become a pathological structure, as during the early stages of spore revival (see the introduction). We show here that DisA limits the processing of a reversed fork by the RuvAB-RecU resolvasome (Figures 3 and 5), and accordingly, it partially reduces chromosomal breakage of unperturbed growing cells (Figures $2 \mathrm{~F}$ and 7 ).

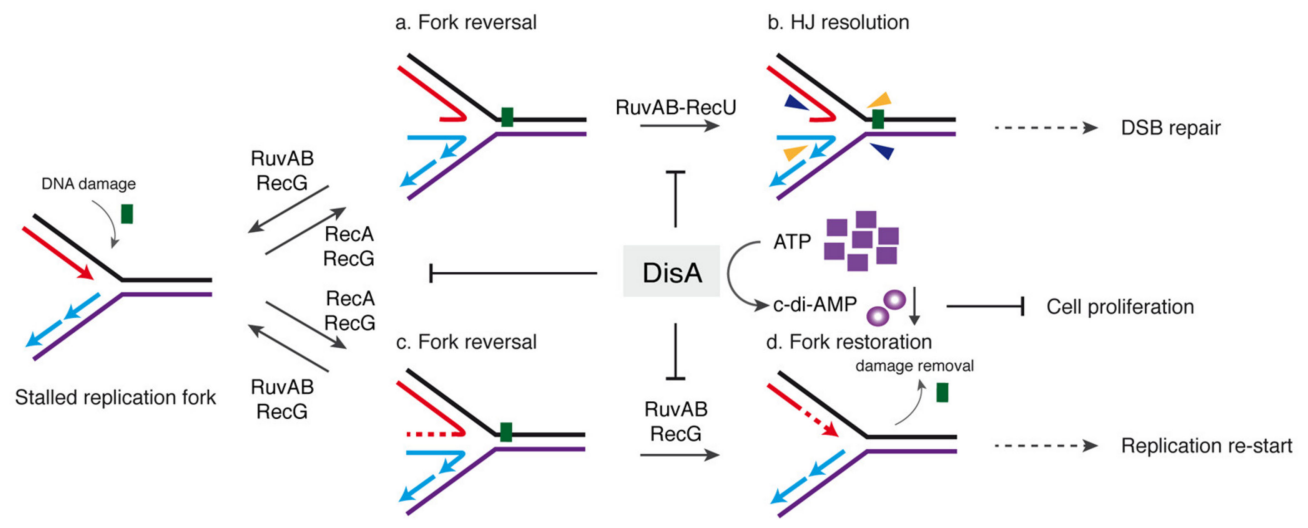

Figure 7. Proposed DisA mode of action in the presence of RecU and RuvAB. An unrepaired DNA lesion on the leading-strand template (green square) causes blockage of replication fork movement. Fork reversal by the RecA or RecG remodeler may form an HJ DNA structure and this process is inhibited by DisA. In the upper panel, DisA suppresses fork reversal and HJ resolution by the RuvAB-RecU resolvasome (a,b), avoiding the formation of a one-ended DSB. In the lower panel, DNA synthesis extends the regressed fork to convert it into an HJ-like structure, and fork restoration is catalyzed either by RuvAB or RecG (c,d), followed by damage removal by specific repair mechanisms. This process is also downregulated by DisA. DisA bound to HJ DNA decreases c-di-AMP synthesis, indirectly increasing (p)ppGpp synthesis and inhibiting cell proliferation.

From the results presented here and in previous reports, we propose that, in response to a 4NQO- or MMS-induced insult, the replisome disengages from the DNA [58], exposing a stalled fork to be remodeled, with SsbA (counterpart of $\mathrm{SSB}_{E c o}$ ) coating the ssDNA lesion-containing gap region $[5,59]$. Second, with the help of mediators and modulators, RecA forms a nucleoprotein filament [60]. This nucleoprotein filament induces the SOS response (increasing RecA and RuvAB levels), and a membrane stress increases DisA and RecU levels. Third, dynamic DisA, which scans for its cognate target site [17], interacts with the RecA nucleoprotein filament and with its cognate site (a stalled or reversed fork) and pauses there, also reducing RecA dynamics [18]. Static DisA bound to a branched intermediate (or the static RecA nucleoprotein filament) might prevent the degradation of the nascent strands of the reversed fork (Figure 2F). Fourth, RecG (or RecA) bound to a stalled fork could convert it into a reversed fork (see above). Fifth, RuvB interacts with DisA and RecU at reversed forks [36,57]. Sixth, DisA bound to a reversed fork partially suppresses c-di-AMP synthesis, and RuvAB or RecU, upon binding to a DisA-HJ DNA complex, synergistically block c-di-AMP synthesis (Figure 6). Seventh, low c-di-AMP levels increase the production of (p)ppGpp, which in turn directly inhibits the DNA primase and indirectly inhibits cell proliferation $[25,26]$, to avoid the uncoupling of the cell cycle. Eighth, in the absence of DisA, RuvAB bound to HJ DNA could branch migrate it until the RecU cognate site is exposed, and thereby assists RecU to cleave the HJ structure [41]. Ninth, DisA, acting as an "emergency brake", limits/delays RuvAB-mediated fork remodeling and RecU-mediated HJ cleavage to stabilize a stalled or reversed fork, to prevent nascent 
strand degradation and genome instability. In other words, DisA bound to a reversed fork protects the extruded nascent strands from an unscheduled cleavage by the RuvAB-RecU resolvasome until the lesion is circumvented (Figures 2, 4 and 7a,b). When the $3^{\prime}$-end of the nascent leading-strand is elongated, using it as a template the nascent lagging-strand, RuvAB (or RecG) could remodel the HJ structure back to a fork structure, and this process is also modulated by DisA (Figure 7c,d). Finally, the offending lesion is removed from duplex DNA, the fork reconstituted, RuvAB and RecU disassembled from the DNA, and replication restarted. DisA recovers its dynamic behavior and catalyzes c-di-AMP synthesis in order to reactivate cell proliferation (Figure 7).

In summary, our present findings reveal that DisA, recruited by RecA (see the introduction), may protect a stalled or reversed fork from degradation as proposed for the eukaryotic mediator BRCA2 and/or Rad51 itself [9]. DisA, as a guardian of genome integrity, provides a quality control to prevent a physiological reversed fork from becoming a pathological one during spore revival by interacting with and/or limiting RuvAB and RecU (this work) or RecG activities [27]. Moreover, a variation in the order of protein recruitment might generate different outcomes to increase survival under different conditions (e.g., sporulating cells and reviving spores vs. exponentially growing cells). The presence of DisA in non-spore-forming bacteria (e.g., Mycobacterium tuberculosis) also provides a conceptual framework for future studies exploring the DisA regulatory balance to overcome replicative stress and its broader role in genome stability in bacteria.

\section{Materials and Methods}

\subsection{Bacterial Strain and Plasmids}

E. coli BL21(DE3) cells bearing pLysS and pET-derived plasmids were used for protein overexpression. XL1-Blue cells were used for cloning and plasmid amplification, and BTH101 cells bearing plasmids pUT18 and pUT18C (to generate fusions at the N- and Ctermini of the T18 domain, respectively), pKNT25 and pKT25 (to generate fusions at the Nand C-termini of the T25 domain, respectively), and its derivatives were used for bacterial two-hybrid analyses. E. coli plasmids pCB875, pCB568, pCB632, pCB1080, and pCB1081 were used to over-express and purify the DisA, RecU, RuvA, DisA D77N, and DisA $\triangle \mathrm{C} 290$ proteins, respectively $[21,24,41,46]$. B. subtilis BG214 cells bearing the pCB737-borne ruvB gene were used to over-express and purify RuvB [61].

\subsection{Protein-Protein Interaction Assays}

In vivo protein-protein interaction was assayed using the bacterial adenylate cyclasebased two-hybrid (BACTH) technique as described [24]. The plasmid-borne DisA (or DisA $\triangle \mathrm{C} 290$ (which lacks the C-terminal 70 residues)) fusions to the T18 or T25 catalytic domain of the Bordetella adenylate cyclase, either at the N- (DisA-T18 and DisA-T25) or C-terminus (T18-DisA or T25-DisA), were pairwise co-transformed into the reporter BTH101 strain with plasmid-borne RuvA, RuvB, or RecU fusions, also to the T18 or T25 catalytic domain, either at the N-or the C-terminus. The empty vectors or the pKT25-Zip and pUT18C-Zip vectors were co-transformed into the reporter strain as negative and positive controls, respectively. Serial dilutions were spotted onto LB plates supplemented with ampicillin, kanamycin, streptomycin, $0.5 \mathrm{mM} \mathrm{IPTG}$, and 10\% X-Gal. The plates were then incubated at $25^{\circ} \mathrm{C}$ for 3-4 days and photographed. Each co-transformation was performed at least in triplicate and a representative result is shown.

\subsection{Pulse-Field Gel Electrophoresis}

Cultures of B. subtilis BG214 (trpCE metA5 amyE1 ytsJ1 rsbV37 xre1 $x k d \mathrm{~A} 1$ att ${ }^{\mathrm{SPB}}$ $a_{t}{ }^{\mathrm{ICEBs} 1}$ ) and its isogenic derivatives lacking DisA (BG1221, $\Delta$ disA) or RecU (BG855, $\Delta r e c U)[21,61]$ were grown at $37^{\circ} \mathrm{C}$ in $\mathrm{LB}$ medium to an $\mathrm{OD}_{560}$ of 0.6 . Then, cells were treated or not with $10 \mathrm{mM}$ MMS for $20 \mathrm{~min}$. A volume of culture corresponding to $\sim 2 \times 10^{8}$ cells was then centrifuged $\left(14,000 \mathrm{rpm}, 5 \mathrm{~min}\right.$ at $\left.4{ }^{\circ} \mathrm{C}\right)$ and washed twice with $1.5 \mathrm{~mL}$ of TEN buffer ( $50 \mathrm{mM}$ Tris-HCl pH 8, $50 \mathrm{mM}$ EDTA, $100 \mathrm{mM} \mathrm{NaCl}$ ). Finally, the cell 
pellet was resuspended in $0.1 \mathrm{~mL}$ of TEN buffer containing $2 \mu \mathrm{g}$ of lysozyme, mixed with an equal volume of $2 \%$ Certified Low Melt Agarose (Bio-Rad, Hercules, CA, USA) brought to $55^{\circ} \mathrm{C}$, dispensed into wells of disposable molds (Bio-Rad), and solidified. The agarose plugs were incubated for $20 \mathrm{~h}$ at $50{ }^{\circ} \mathrm{C}$ in NDS buffer $(10 \mathrm{mM}$ Tris- $\mathrm{HCl}$ pH 8.0, 0.4 M EDTA $\mathrm{pH} 8.0,1 \% \mathrm{~N}$-lauryl sarcosine) with $0.35 \mathrm{mg} / \mathrm{mL}$ proteinase $\mathrm{K}$. After completion of the incubation, the lysis buffer was replaced with TE buffer and the plugs were stored at $4{ }^{\circ} \mathrm{C}$ until used. A CHEF-DR II pulsed-field gel electrophoresis system (Bio-Rad) was used to resolve the DNA. Running conditions were $20 \mathrm{~h}, 7^{\circ} \mathrm{C}, 5 \mathrm{~V} / \mathrm{cm}$, with a pulse time of $5-80 \mathrm{~s}$. The $1 \%$ agarose gel was stained with ethidium bromide and photographed. The densitometric analysis of the lanes was performed using the Image Lab software (Bio-Rad). The percentage of chromosomal fragmentation was calculated as the signal between 610 and $48 \mathrm{~Kb}$ in the lane, divided by the combined signal of the lane plus well. The experiment was performed three times, and results are plotted relative to the value obtained with the $w t$ in the untreated condition. $t$-tests were applied to analyze the statistical significance of the data.

\subsection{DNA Substrates}

The nucleotide (nt) sequences of the oligonucleotides used are indicated in the $5^{\prime} \rightarrow 3^{\prime}$ polarity: J3-1, CGCAAGCGACAGGAACCTCGAGAAGCTTCCGGTAGCAGCCT GAGCGGTGGTTGAATTCCTCGAGGTTCCTGTCGCTTGCG; J3-2, CGCAGCGACAGG AACCTCGAGGAATTCAACCACCGCTCAACTCAACTGCAGTCTGACTCGAGGTTC CTGTCGCTTGCG; J3-3, CGCAAGCGACAGGAACCTCGAGTCTAGACTGCAGTTGA GTCCTTGCTAGGACGGATCCCTCAGGTTCCTGTCGCTTGCG; J3-4, CGCAAGCGAC AGGAACCTCGAGGGATCCGTCCTAGCAAGGGGCTGCTACCGGAAGCTTCTCGA GGTTCCTGTCGCTTGCG; J3-5, CGCAAGCG ACAGGAACCTCGAGTCTAGACTGCA GTTGAGTTGAGCGGTGGTTGAATTCCTCGAGT TCCTGTCGCTTGCG; J170, CTAGA GACGCTGCCGAATTCTGGCTTGGATCTGATGCTGTCTAGAGGCCTCCACTATGA AATCGCTGCA; J173, CCGGGCTGCAGAGCTCATAGATCGATAGTCTCTAGACAGC ATCAGATCCAAGCCAGAATTCGGCAGCGTCT; J345, GCGATTTCATAGTGGAGGC CTCTAGACAGCACGCCGTTGAATGGGCGGATGCTAATTACTATC TC; J346, GAGA TAGTAATTAGCATCCGCCCATTCAACGGCGTGCTGTCTAGAGACTATCGATCTAT GAGCTCTGCAGC; 170, AGACGCTGCCGAATTCTGGCTTGGATCTGATGCTGTCTA GAGGCCTCCACTATGAAATCG; 173, AGCTCATAGATCGATAGTCTCTAGACAGC ATCAGATCCAAGCCAGAATTCGGCAGCGTCT; 171, CGATTTCATAGTGGAGGCCT CTAGACAGCA; 172, TGCTGTCTAGAGACTATCGATCTATGAGCT; 171-15, CGATTT CATAGTGGA and 172-15, ATCGATCTATGAGCT. The dsDNA was assembled by annealing J3-2 and J3-5 (80-bp); fixed HJ (HJ-J3 DNA) by annealing J3-1, J3-2, J3-3, and J3-4; mobile HJ (HJ-J4 DNA) by annealing J170, J173, J345, and J346; flayed by annealing 170 and 173; forked-Lag by annealing 170, 173, 171, and 172-15; and forked-Lead by annealing $170,173,171-15$, and 172 . The ssDNA concentrations were measured using the extinction coefficient of $1.54 \times 10^{-4} \mathrm{M}^{-1} \mathrm{~cm}^{-1}$ at $260 \mathrm{~nm}$, and the concentrations of DNA substrates are expressed as moles of DNA molecules or moles of nucleotides as indicated. Annealing was performed by mixing the appropriate oligonucleotides in $50 \mathrm{mM}$ phosphate buffer $\mathrm{pH} 7.5$, heating for $5 \mathrm{~min}$ at $100^{\circ} \mathrm{C}$, and then slowly cooling. The annealed products were gel purified as described previously, dialyzed against buffer A (50 mM Tris- $\mathrm{HCl}$ ( $\mathrm{pH} 7.0$ ), $5 \%$ glycerol, $1 \mathrm{mM}$ DTT), and stored at $4{ }^{\circ} \mathrm{C}$ [42].

\subsection{Protein Purification}

The RecU, RuvA, RuvB, DisA, DisA D77N, and DisA $\Delta$ C290 proteins were purified as described $[18,23,24,41,46]$. The proteins were $>95 \%$ pure based on staining after SDS-PAGE, and partial proteolysis and MALDI-TOF analysis. RuvA was free of the RuvA $A_{E c o}$ protein. The molar extinction coefficients for DisA, RecU, RuvA, and RuvB were calculated as 22,350, 27,850,11,900, and 13,400 $\mathrm{M}^{-1} \mathrm{~cm}^{-1}$ at $280 \mathrm{~nm}$, as described [57]. The concentrations of RecU, RuvA, RuvB, and DisA (and its DAC active site [DisA D77N] or DNA-binding 
domain [DisA $\Delta$ C290] mutant) are expressed as moles of dimers, tetramers, hexamers, and octamers, respectively.

\subsection{ATPase Activity and c-di-AMP Synthesis}

The ATP hydrolysis activity of the RuvAB protein was assayed via an ATP/NADHcoupled spectrophotometric enzymatic assay. Assays were done in buffer B (50 mM Tris- $\mathrm{HCl} \mathrm{pH} 7.5,80 \mathrm{mM} \mathrm{NaCl}, 10 \mathrm{mM} \mathrm{Mg}\left(\mathrm{CH}_{3} \mathrm{COO}\right)_{2}, 50 \mu \mathrm{g} / \mathrm{mL}$ bovine serum albumin (BSA), $1 \mathrm{mM}$ DTT, 5\% glycerol) containing $5 \mathrm{mM}$ ATP and an ATP regeneration system $(620 \mu \mathrm{M}$ NADH, $100 \mathrm{U} / \mathrm{mL}$ of lactate dehydrogenase, $500 \mathrm{U} / \mathrm{mL}$ pyruvate kinase, and $2.5 \mathrm{mM}$ phosphoenolpyruvate) for $30 \mathrm{~min}$ at $37^{\circ} \mathrm{C}$, as described.

The order of addition of DNA effectors (circular 3199-nt pGEM3 Zf (+) ssDNA or HJ DNA [10 $\mu \mathrm{M}$ in $\mathrm{nt}]$ ) and purified proteins is indicated in the text. The data obtained from the rate of NADH absorbance decrease at $340 \mathrm{~nm}$ is proportional to the rate of ADP production, and it is plotted as a function of time. As reported [59], the rate of ATP hydrolysis $\left(\mathrm{K}_{\text {cat }}\right)$ was derived from the slope of the linear part of the curves. $t$-tests were applied to analyze the statistical significance of the data.

c-di-AMP formation was analyzed using thin-layer chromatography (TLC) and $\left[\alpha-{ }^{32} \mathrm{P}\right]-$ ATP as described [22,23]. Reactions were performed at $37{ }^{\circ} \mathrm{C}$ using a range of protein concentrations, in buffer D (50 mM Tris- $\mathrm{HCl}$ pH 7.5, $50 \mathrm{mM} \mathrm{NaCl}, 1 \mathrm{mM}$ DTT, $0.5 \mu \mathrm{g} / \mathrm{mL}$ BSA, $0.1 \%$ Triton, $5 \%$ glycerol) containing $100 \mu \mathrm{M}$ ATP (at a ratio of 1:2000 [ $\left.\alpha^{32} \mathrm{P}\right]$-ATP:ATP) and the indicated $\mathrm{MgCl}_{2}$ and $\mathrm{HJ}$ DNA concentration. After 30 min of incubation, the reaction was chelated by adding $50 \mathrm{mM}$ EDTA, and $2 \mu \mathrm{L}$ of each reaction were spotted onto $20 \times 20 \mathrm{~cm}$ TLC polyethyleneimine cellulose plates and run for about $2 \mathrm{~h}$ in a TLC chamber containing running buffer $\mathrm{E}\left[1: 1(v / v) 1.5 \mathrm{M} \mathrm{KH}_{2} \mathrm{PO}_{4}(\mathrm{pH} 3.6)\right.$ and 70\% ammonium sulfate]. Dried TLC plates were analyzed by phosphorimaging and spots were quantified using ImageJ (NIH). $t$-tests were applied to analyze the statistical significance of the data.

\subsection{DNA Binding, HJ Branch Migration, and Cleavage Assays}

DNA binding was assayed by EMSA using different $\left[\gamma^{32} \mathrm{P}\right]$-labelled DNA substrates (0.2 $\mathrm{nM}$ in molecules). The radiolabeled strand is indicated with an asterisk. The binding was performed in buffer C (50 mM Tris- $\mathrm{HCl} \mathrm{pH}$ 7.5, $50 \mathrm{mM} \mathrm{NaCl}, 1 \mathrm{mM}$ DTT, $0.05 \mathrm{mg} / \mathrm{mL}$ BSA, $5 \%$ glycerol) containing 1 or $10 \mathrm{mM} \mathrm{MgCl}_{2}$ or $5 \mathrm{mM}$ EDTA and when indicated a nucleotide cofactor. Reactions were incubated for $15 \mathrm{~min}$ at $37^{\circ} \mathrm{C}$. Prior to loading, $0.2 \%$ glutaraldehyde was added to stabilize the complexes. Protein-DNA complexes were separated using 6\% PAGE in TAE buffer and visualized by autoradiography. Autoradiography films were scanned, and the ImageJ software (NIH, Bethesda, MD, USA) was used to determine the signal from each band and obtain the apparent binding constant $\left(\mathrm{K}_{\text {Dapp }}\right)$ values at the protein concentration that gives $50 \%$ of DNA-protein complexes. $t$-tests were applied to analyze the statistical significance of the data.

The reaction conditions for DNase I footprint experiments were the same as for EMSA. DNase I treatment was performed as described [62]. The samples were resuspended in loading buffer [80\% (v/v) formamide, $10 \mathrm{mM} \mathrm{NaOH}, 1 \mathrm{mM}$ EDTA, $0.1 \%(v / v)$ bromophenol blue, and $0.1 \%(v / v)$ xylene cyanol], separated in $15 \%$ denaturing PAGE (dPAGE), and autoradiographed. For the size control marker, ladders obtained with the chemical sequencing reaction $(G+A)$ on the same DNA fragments were used.

Cleavage of HJ-J3 DNA (labeled on arm 1) at the indicated concentrations of RecU was assayed for $30 \mathrm{~min}$ at $37^{\circ} \mathrm{C}$ in buffer $\mathrm{C}$ containing $10 \mathrm{mM} \mathrm{MgCl}$. When indicated, increasing concentrations of DisA were added. After deproteinization by the addition of one-fifth volume of stop mix (5\% SDS, $100 \mathrm{mM}$ EDTA, $5 \mathrm{mg} / \mathrm{mL}$ proteinase $\mathrm{K}$ ) and further incubation for $10 \mathrm{~min}$ at $37{ }^{\circ} \mathrm{C}$, the products of the cleavage were analyzed by $15 \%$ denaturing PAGE and autoradiography. $t$-tests were applied to analyze the statistical significance of the data.

In a standard branch migration assay, $\left[\gamma^{32} \mathrm{P}\right]$-labelled HJ-J4 DNA (0.1 nM in molecules) was incubated with RuvAB in buffer $\mathrm{C}$ containing $10 \mathrm{mM} \mathrm{MgCl}_{2}$ and $5 \mathrm{mM}$ ATP for $30 \mathrm{~min}$ 
at $37^{\circ} \mathrm{C}$. When indicated, increasing concentrations of DisA were added. Reactions were terminated by adding one-fifth volume of stop mix (5\% SDS, $100 \mathrm{mM}$ EDTA, $5 \mathrm{mg} / \mathrm{mL}$ proteinase K) and further incubation for $10 \mathrm{~min}$ at $37{ }^{\circ} \mathrm{C}$ to deproteinize the sample. Unwound products were analyzed by 6\% PAGE in TAE buffer and phosphorimaging. Signals of substrates and products were quantified with the ImageJ software (NIH). $t$-tests were applied to analyze the statistical significance of the data.

Supplementary Materials: The following are available online at https: / / www.mdpi.com/article / 10.3390 /ijms222111323/s1, Figure S1: Affinity of DisA for different DNA substrates, Figure S2: DisA DAC activity requirements; Figure S3: Extent of spontaneous chromosomal fragmentation in the $\Delta r e c U$ context.

Author Contributions: Conceptualization, S.A. and J.C.A.; formal analysis, validation and investigation, C.G., R.T. and B.C.; data curation, C.G., R.T., B.C., S.A. and J.C.A.; writing-original draft preparation, S.A. and J.C.A.; writing-review and editing, R.T., B.C., S.A. and J.C.A.; supervision, S.A. and J.C.A. All authors have read and agreed to the published version of the manuscript.

Funding: This research was funded by Ministerio de Ciencia e Innovación/Agencia Estatal de Investigación (MCeI/AEI)/FEDER, EU). Grant No. PGC2018-097054-B-I00 to S.A and J.C.A.

Institutional Review Board Statement: Not applicable.

Informed Consent Statement: Not applicable.

Data Availability Statement: The datasets analyzed or generated during the study are avalaible upon request.

Acknowledgments: We are grateful to Karl-Peter Hopfner and Gregor Witte for the communication of unpublished results, and to Cristina Cañas for her contribution in the early steps of the project. C.G. was a recipient of a Fellowship of the Ministerio de Economia y Competividad (MINECO) (BES-2010-037211), R.T. was a PhD fellow of La Caixa Foundation International Fellowship Program (La Caixa/CNB) and B.C. thanks the Programa Centros de Excelencia Severo Ochoa-CNB for the fellowship.

Conflicts of Interest: The authors declare no conflict of interest. The funders had no role in the design of the study; in the collection, analyses, or interpretation of data; in the writing of the manuscript, or in the decision to publish the results.

\section{References}

1. Cox, M.M.; Goodman, M.F.; Kreuzer, K.N.; Sherratt, D.J.; Sandler, S.J.; Marians, K.J. The importance of repairing stalled replication forks. Nature 2000, 404, 37-41. [CrossRef]

2. Branzei, D.; Foiani, M. Maintaining genome stability at the replication fork. Nat. Rev. Mol. Cell Biol. 2010, 11, 208-219. [CrossRef]

3. Ciccia, A.; Elledge, S.J. The DNA damage response: Making it safe to play with knives. Mol. Cell 2010, 40, 179-204. [CrossRef]

4. Zeman, M.K.; Cimprich, K.A. Causes and consequences of replication stress. Nat. Cell Biol. 2014, 16, 2-9. [CrossRef] [PubMed]

5. Kowalczykowski, S.C. An Overview of the molecular mechanisms of recombinational DNA repair. Cold Spring Harb. Perspect Biol. 2015, 7, a016410. [CrossRef]

6. Neelsen, K.J.; Lopes, M. Replication fork reversal in eukaryotes: From dead end to dynamic response. Nat. Rev. Mol. Cell Biol. 2015, 16, 207-220. [CrossRef] [PubMed]

7. Marians, K.J. Lesion bypass and the reactivation of stalled replication forks. Annu. Rev. Biochem. 2018, 87, 217-238. [CrossRef] [PubMed]

8. Michel, B.; Sinha, A.K.; Leach, D.R.F. Replication fork breakage and restart in Escherichia coli. Microbiol. Mol. Biol. Rev. 2018, 82, e00013-18. [CrossRef]

9. Thakar, T.; Moldovan, G.L. The emerging determinants of replication fork stability. Nucleic. Acids Res. 2021, 49, 7224-7238. [CrossRef]

10. Seigneur, M.; Bidnenko, V.; Ehrlich, S.D.; Michel, B. RuvAB acts at arrested replication forks. Cell 1998, 95, 419-430. [CrossRef]

11. Berti, M.; Cortez, D.; Lopes, M. The plasticity of DNA replication forks in response to clinically relevant genotoxic stress. Nat. Rev. Mol. Cell Biol. 2020, 21, 633-651. [CrossRef]

12. Vlasic, I.; Mertens, R.; Seco, E.M.; Carrasco, B.; Ayora, S.; Reitz, G.; Commichau, F.M.; Alonso, J.C.; Moeller, R. Bacillus subtilis RecA and its accessory factors, RecF, RecO, RecR and RecX, are required for spore resistance to DNA double-strand break. Nucleic. Acids Res. 2014, 42, 2295-2307. [CrossRef] [PubMed] 
13. Raguse, M.; Torres, R.; Seco, E.M.; Gándara, C.; Ayora, S.; Moeller, R.; Alonso, J.C. Bacillus subtilis DisA helps to circumvent replicative stress during spore revival. DNA Repair (Amst) 2017, 59, 57-68. [CrossRef]

14. Nicolas, P.; Mader, U.; Dervyn, E.; Rochat, T.; Leduc, A.; Pigeonneau, N.; Bidnenko, E.; Marchadier, E.; Hoebeke, M.; Aymerich, S.; et al. Condition-dependent transcriptome reveals high-level regulatory architecture in Bacillus subtilis. Science 2012, 335, $1103-1106$. [CrossRef] [PubMed]

15. Sinai, L.; Rosenberg, A.; Smith, Y.; Segev, E.; Ben-Yehuda, S. The molecular timeline of a reviving bacterial spore. Mol. Cell 2015, 57, 695-707. [CrossRef] [PubMed]

16. Corrigan, R.M.; Grundling, A. Cyclic di-AMP: Another second messenger enters the fray. Nat. Rev. Microbiol. 2013, 11, 513-524. [CrossRef] [PubMed]

17. Bejerano-Sagie, M.; Oppenheimer-Shaanan, Y.; Berlatzky, I.; Rouvinski, A.; Meyerovich, M.; Ben-Yehuda, S. A checkpoint protein that scans the chromosome for damage at the start of sporulation in Bacillus subtilis. Cell 2006, 125, 679-690. [CrossRef]

18. Torres, R.; Carrasco, B.; Gándara, C.; Baidya, A.K.; Ben-Yehuda, S.; Alonso, J.C. Bacillus subtilis DisA regulates RecA-mediated DNA strand exchange. Nucleic. Acids Res. 2019, 47, 5141-5154. [CrossRef]

19. Oppenheimer-Shaanan, Y.; Wexselblatt, E.; Katzhendler, J.; Yavin, E.; Ben-Yehuda, S. c-di-AMP reports DNA integrity during sporulation in Bacillus subtilis. EMBO Rep. 2011, 12, 594-601. [CrossRef]

20. Merrick, C.J.; Jackson, D.; Diffley, J.F. Visualization of altered replication dynamics after DNA damage in human cells. J. Biol. Chem. 2004, 279, 20067-20075. [CrossRef]

21. Gándara, C.; Alonso, J.C. DisA and c-di-AMP act at the intersection between DNA-damage response and stress homeostasis in exponentially growing Bacillus subtilis cells. DNA Repair (Amst) 2015, 27, 1-8. [CrossRef]

22. Witte, G.; Hartung, S.; Buttner, K.; Hopfner, K.P. Structural biochemistry of a bacterial checkpoint protein reveals diadenylate cyclase activity regulated by DNA recombination intermediates. Mol. Cell 2008, 30, 167-178. [CrossRef] [PubMed]

23. Gándara, C.; de Lucena, D.K.C.; Torres, R.; Serrano, E.; Altenburger, S.; Graumann, P.L.; Alonso, J.C. Activity and in vivo dynamics of Bacillus subtilis DisA are affected by RadA/Sms and by Holliday junction-processing proteins. DNA Repair (Amst) 2017, 55, 17-30. [CrossRef]

24. Torres, R.; Serrano, E.; Tramm, K.; Alonso, J.C. Bacillus subtilis RadA/Sms contributes to chromosomal transformation and DNA repair in concert with RecA and circumvents replicative stress in concert with DisA. DNA Repair (Amst) 2019, 77, 45-57. [CrossRef] [PubMed]

25. Kruger, L.; Herzberg, C.; Wicke, D.; Bahre, H.; Heidemann, J.L.; Dickmanns, A.; Schmitt, K.; Ficner, R.; Stulke, J. A meet-up of two second messengers: The c-di-AMP receptor DarB controls (p)ppGpp synthesis in Bacillus subtilis. Nat. Commun. 2021, 12, 1210. [CrossRef] [PubMed]

26. Wang, J.D.; Sanders, G.M.; Grossman, A.D. Nutritional control of elongation of DNA replication by (p)ppGpp. Cell 2007, 128, 865-875. [CrossRef] [PubMed]

27. Torres, R.; Gándara, C.; Carrasco, B.; Baquedano, I.; Ayora, S.; Alonso, J.C. DisA limits RecG activities at stalled or reversed replication forks. Cells 2021, 10, 1357. [CrossRef]

28. Robu, M.E.; Inman, R.B.; Cox, M.M. RecA protein promotes the regression of stalled replication forks in vitro. Proc. Natl. Acad. Sci. USA 2001, 98, 8211-8218. [CrossRef]

29. McGlynn, P.; Mahdi, A.A.; Lloyd, R.G. Characterisation of the catalytically active form of RecG helicase. Nucleic Acids Res. 2000, 28, 2324-2332. [CrossRef]

30. McGlynn, P.; Lloyd, R.G.; Marians, K.J. Formation of Holliday junctions by regression of nascent DNA in intermediates containing stalled replication forks: RecG stimulates regression even when the DNA is negatively supercoiled. Proc. Natl. Acad. Sci. USA 2001, 98, 8235-8240. [CrossRef]

31. Abd Wahab, S.; Choi, M.; Bianco, P.R. Characterization of the ATPase activity of RecG and RuvAB proteins on model fork structures reveals insight into stalled DNA replication fork repair. J. Biol. Chem. 2013, 288, 26397-26409. [CrossRef]

32. Bagchi, D.; Manosas, M.; Zhang, W.; Manthei, K.A.; Hodeib, S.; Ducos, B.; Keck, J.L.; Croquette, V. Single molecule kinetics uncover roles for E. coli RecQ DNA helicase domains and interaction with SSB. Nucleic. Acids Res. 2018, 46, 8500-8515. [CrossRef]

33. Torres, R.; Alonso, J.C. Bacillus subtilis RecA, DisA and RadA/Sms interplay prevents replication stress by regulating fork remodeling. Front. Microbiol. 2021. In press.

34. Au, N.; Kuester-Schoeck, E.; Mandava, V.; Bothwell, L.E.; Canny, S.P.; Chachu, K.; Colavito, S.A.; Fuller, S.N.; Groban, E.S.; Hensley, L.A.; et al. Genetic composition of the Bacillus subtilis SOS system. J. Bacteriol. 2005, 187, 7655-7666. [CrossRef]

35. Eiamphungporn, W.; Helmann, J.D. The Bacillus subtilis $\sigma^{\mathrm{M}}$ regulon and its contribution to cell envelope stress responses. Mol. Microbiol. 2008, 67, 830-848. [CrossRef]

36. Cañas, C.; Carrasco, B.; Garcia-Tirado, E.; Rafferty, J.B.; Alonso, J.C.; Ayora, S. The stalk region of the RecU resolvase is essential for Holliday junction recognition and distortion. J. Mol. Biol. 2011, 410, 39-49. [CrossRef]

37. West, S.C. Processing of recombination intermediates by the RuvABC proteins. Annu. Rev. Genet. 1997, 31, 213-244. [CrossRef] [PubMed]

38. Hargreaves, D.; Rice, D.W.; Sedelnikova, S.E.; Artymiuk, P.J.; Lloyd, R.G.; Rafferty, J.B. Crystal structure of E. coli RuvA with bound DNA Holliday junction at 6 Å resolution. Nat. Struct. Biol. 1998, 5, 441-446. [CrossRef] [PubMed]

39. van Gool, A.J.; Shah, R.; Mezard, C.; West, S.C. Functional interactions between the Holliday junction resolvase and the branch migration motor of Escherichia coli. EMBO J. 1998, 17, 1838-1845. [CrossRef] [PubMed] 
40. Nishino, T.; Ariyoshi, M.; Iwasaki, H.; Shinagawa, H.; Morikawa, K. Functional analyses of the domain structure in the Holliday junction binding protein RuvA. Structure 1998, 6, 11-21. [CrossRef]

41. Cañas, C.; Suzuki, Y.; Marchisone, C.; Carrasco, B.; Freire-Beneitez, V.; Takeyasu, K.; Alonso, J.C.; Ayora, S. Interaction of branch migration translocases with the Holliday junction-resolving enzyme and their implications in Holliday junction resolution. J. Biol. Chem. 2014, 289, 17634-17646. [CrossRef] [PubMed]

42. McGregor, N.; Ayora, S.; Sedelnikova, S.; Carrasco, B.; Alonso, J.C.; Thaw, P.; Rafferty, J. The structure of Bacillus subtilis RecU Holliday junction resolvase and its role in substrate selection and sequence-specific cleavage. Structure 2005, 13, 1341-1351. [CrossRef] [PubMed]

43. Khavnekar, S.; Dantu, S.C.; Sedelnikova, S.; Ayora, S.; Rafferty, J.; Kale, A. Structural insights into dynamics of RecU-HJ complex formation elucidates key role of NTR and stalk region toward formation of reactive state. Nucleic. Acids Res. 2017, 45, 975-986. [CrossRef] [PubMed]

44. Amit, R.; Gileadi, O.; Stavans, J. Direct observation of RuvAB-catalyzed branch migration of single Holliday junctions. Proc. Natl. Acad. Sci. USA 2004, 101, 11605-11610. [CrossRef] [PubMed]

45. Dawid, A.; Croquette, V.; Grigoriev, M.; Heslot, F. Single-molecule study of RuvAB-mediated Holliday-junction migration. Proc. Natl. Acad. Sci. USA 2004, 101, 11611-11616. [CrossRef]

46. Ayora, S.; Carrasco, B.; Doncel, E.; Lurz, R.; Alonso, J.C. Bacillus subtilis RecU protein cleaves Holliday junctions and anneals single-stranded DNA. Proc. Natl. Acad. Sci. USA 2004, 101, 452-457. [CrossRef] [PubMed]

47. Carrasco, B.; Cañas, C.; Sharples, G.J.; Alonso, J.C.; Ayora, S. The N-terminal region of the RecU holliday junction resolvase is essential for homologous recombination. J. Mol. Biol. 2009, 390, 1-9. [CrossRef]

48. Suzuki, Y.; Endo, M.; Cañas, C.; Ayora, S.; Alonso, J.C.; Sugiyama, H.; Takeyasu, K. Direct analysis of Holliday junction resolving enzyme in a DNA origami nanostructure. Nucleic. Acids Res. 2014, 42, 7421-7428. [CrossRef]

49. Parsons, C.A.; Tsaneva, I.; Lloyd, R.G.; West, S.C. Interaction of Escherichia coli RuvA and RuvB proteins with synthetic Holliday junctions. Proc. Natl. Acad. Sci. USA 1992, 89, 5452-5456. [CrossRef]

50. Ingleston, S.M.; Dickman, M.J.; Grasby, J.A.; Hornby, D.P.; Sharples, G.J.; Lloyd, R.G. Holliday junction binding and processing by the RuvA protein of Mycoplasma pneumoniae. Eur. J. Biochem. 2002, 269, 1525-1533. [CrossRef]

51. Panyutin, I.G.; Biswas, I.; Hsieh, P. A pivotal role for the structure of the Holliday junction in DNA branch migration. EMBO J. 1995, 14, 1819-1826. [CrossRef] [PubMed]

52. Hiom, K.; West, S.C. Branch migration during homologous recombination: Assembly of a RuvAB-Holliday junction complex in vitro. Cell 1995, 80, 787-793. [CrossRef]

53. Khan, S.R.; Kuzminov, A. Trapping and breaking of in vivo nicked DNA during pulsed field gel electrophoresis. Anal. Biochem. 2013, 443, 269-281. [CrossRef] [PubMed]

54. Mawer, J.S.; Leach, D.R. Branch migration prevents DNA loss during double-strand break repair. PLoS Genet. 2014, 10 , e1004485. [CrossRef] [PubMed]

55. Marrione, P.E.; Cox, M.M. RuvB protein-mediated ATP hydrolysis: Functional asymmetry in the RuvB hexamer. Biochemistry 1995, 34, 9809-9818. [CrossRef] [PubMed]

56. Iwasaki, H.; Takahagi, M.; Nakata, A.; Shinagawa, H. Escherichia coli RuvA and RuvB proteins specifically interact with Holliday junctions and promote branch migration. Genes Dev. 1992, 6, 2214-2220. [CrossRef] [PubMed]

57. Carrasco, B.; Ayora, S.; Lurz, R.; Alonso, J.C. Bacillus subtilis RecU Holliday-junction resolvase modulates RecA activities. Nucleic. Acids Res. 2005, 33, 3942-3952. [CrossRef] [PubMed]

58. Mangiameli, S.M.; Merrikh, C.N.; Wiggins, P.A.; Merrikh, H. Transcription leads to pervasive replisome instability in bacteria. eLife 2017, 6. [CrossRef]

59. Yadav, T.; Carrasco, B.; Myers, A.R.; George, N.P.; Keck, J.L.; Alonso, J.C. Genetic recombination in Bacillus subtilis: A division of labor between two single-strand DNA-binding proteins. Nucleic. Acids Res. 2012, 40, 5546-5559. [CrossRef]

60. Carrasco, B.; Yadav, T.; Serrano, E.; Alonso, J.C. Bacillus subtilis RecO and SsbA are crucial for RecA-mediated recombinational DNA repair. Nucleic. Acids Res. 2015, 43, 5984-5997. [CrossRef]

61. Cañas, C.; Carrasco, B.; Ayora, S.; Alonso, J.C. The RecU Holliday junction resolvase acts at early stages of homologous recombination. Nucleic. Acids Res. 2008, 36, 5242-5249. [CrossRef] [PubMed]

62. de la Hoz:, A.B.; Ayora, S.; Sitkiewicz, I.; Fernandez, S.; Pankiewicz, R.; Alonso, J.C.; Ceglowski, P. Plasmid copy-number control and better-than-random segregation genes of pSM19035 share a common regulator. Proc. Natl. Acad. Sci. USA 2000, 97, 728-733. [CrossRef] [PubMed] 